\title{
Extension of N-Confused Porphyrin by an o-Xylene Fragment
}

\section{Piotr J. Chmielewski}

Department of Chemistry, University of Wroctaw, 14 F. Joliot-Curie Street, 50383 Wroctaw, Poland

Email Address: pjc@wchuwr.chem.uni.wroc.pl

\section{Supporting Information}




\section{Table of contents}

Instrumentation. $\quad 3$

Preparation of the precursor $\quad 3$

Syntheses of $N$-alkylated derivatives $\mathbf{3}$ and $\mathbf{4} 3$

Synthesis of 5

Synthesis of $6 \quad 5$

Insertion of the silver(III) ion into $6 \quad 6$

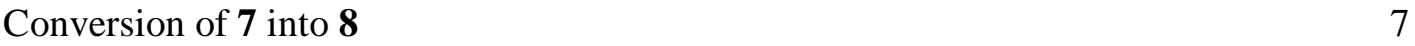

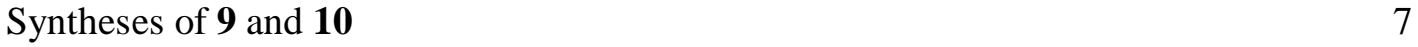

Synthesis of $\mathbf{1 1} \quad 8$

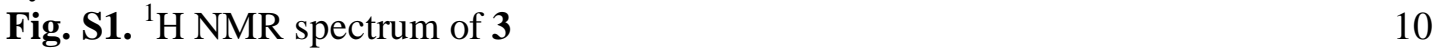

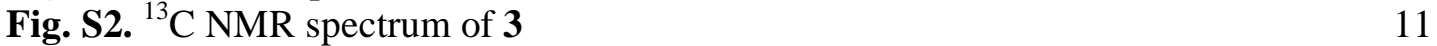

Fig. S3. ${ }^{1} \mathrm{H}$ NMR spectrum of dimer $4 \quad 12$

Fig. S4. ${ }^{13} \mathrm{C}$ NMR spectrum of dimer $4 \quad 13$

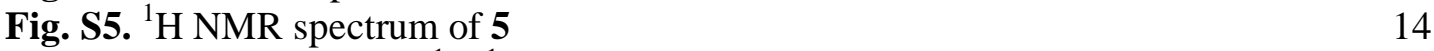

Fig. S6. A fragment of the ${ }^{1} \mathrm{H}-{ }^{1} \mathrm{H}$ COSY spectrum of 5

Fig. S7. ${ }^{1} \mathrm{H}-{ }^{13} \mathrm{C}$ HMQC spectrum of $5 \quad 15$

Fig. S8. ${ }^{1} \mathrm{H}-{ }^{13} \mathrm{C}$ HMBC spectrum of $5 \quad 16$

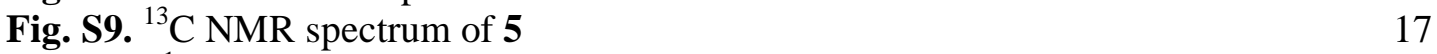

Fig. S10. ${ }^{1}$ H NMR spectrum of $6 \quad 18$

Fig. S11. NOESY spectrum of $6 \quad 19$

Fig. S12. ${ }^{1} \mathrm{H}-{ }^{13} \mathrm{C}$ HMQC spectrum of $6 \quad 20$

Fig. S13. ${ }^{1} \mathrm{H}-{ }^{13} \mathrm{C}$ HMBC spectrum of $6 \quad 21$

Fig. S14. ${ }^{13} \mathrm{C}$ NMR spectrum of $6 \quad 22$

Fig. S15. ${ }^{1} \mathrm{H}$ NMR titration of $\mathbf{5}$ with trifluoroacetic acid , $298 \mathrm{~K}$

Fig. S16. ${ }^{1} \mathrm{H}$ NMR titration of $\mathbf{5}$ with trifluoroacetic acid, $213 \mathrm{~K} \quad 23$

Fig. S17. High-field fragments of the ${ }^{1} \mathrm{H}$ NMR spectra of $6,[6-\mathrm{H}]^{+}$, and $\left[6-\mathrm{H}_{2}\right]^{2+} \quad 24$

Fig. S18. Fragments of the ${ }^{1} \mathrm{H}$ NMR of 6 and $\left[6-\mathrm{H}_{2}\right]^{2+}, 198 \mathrm{~K} \quad 24$

Fig. S19. ${ }^{1} \mathrm{H}$ NMR spectra of 7 and $8 \quad 25$

Fig. S20. ${ }^{1} \mathrm{H}$ NMR spectrum of 926

Fig. S21. ${ }^{1} \mathrm{H}-{ }^{13} \mathrm{C}$ HMBC spectrum of 9

Fig. S22. ${ }^{13} \mathrm{C}$ NMR spectrum of $9 \quad 28$

Fig. S23. ${ }^{1} \mathrm{H}$ NMR spectrum of $\mathbf{1 1} 29$

Fig. S24. ${ }^{13} \mathrm{C}$ NMR spectrum of $\mathbf{1 1} \quad 30$

Fig. S25. A fragment of the ${ }^{1} \mathrm{H}-{ }^{13} \mathrm{C}$ HMQC spectrum of $\mathbf{1 1} 30$

Fig. S26. ${ }^{1} \mathrm{H}-^{13} \mathrm{C}$ HMBC spectrum of $\mathbf{1 1} 31$

Fig. S27. ${ }^{11}$ B NMR spectrum of $\mathbf{1 1} 31$

Fig. S28. Optical spectrum of $\mathbf{4}$ in dichloromethane 32

Fig. S29. Optical spectra of $\mathbf{7}$ and $\mathbf{8} \quad 32$

Fig. S30. Optical spectra of 9 and $\mathbf{1 0} \quad 33$

Fig. S31. Optical spectrum of $\mathbf{1 1} 33$ 
Instrumentation. Absorption spectra were recorded on a diode array Hewlett-Packard 8453 spectrometer. Mass spectra were recorded on an AD-604 spectrometer using the electrospray ionization technique. NMR spectra were recorded on a Bruker Avance 500 spectrometer. The 1D and 2D experiments (COSY, NOESY, HMQC, and HMBC) were performed by means of standard experimental procedures of the Bruker library. The peaks were referenced to the residual $\mathrm{CHCl}_{3}$ resonances in ${ }^{1} \mathrm{H}$ and ${ }^{13} \mathrm{C}$ NMR (7.24 ppm and $77.2 \mathrm{ppm}$, respectively).

Preparation of the precursor. 5,10,15,20-Tetrakis( $p$-tolyl)-2-aza-21-carbaporphyrin 1 was synthesized according to the known procedure (Ref. 3).

Syntheses of $\boldsymbol{N}$-alkylated derivatives 3 and 4 . In a typical synthesis $50 \mathrm{mg}(0.075 \mathrm{mmole})$ of 1 was refluxed for $6 \mathrm{~h}$ in a dry toluene $(30 \mathrm{~mL})$ with $59 \mathrm{mg}(0.225 \mathrm{mmole})$ of $1,2-$ dibromomethylbenzene along with a suspension of $20 \mathrm{mg}$ of $\mathrm{K}_{2} \mathrm{CO}_{3}$ under nitrogen. Flash chromatography on a neutral aluminum oxide column (activity $3^{\circ}$ ) with benzene allows the separation of reaction products 3 (second band) and 4 (third band) from the excess of the alkylating agent and its decomposition products (fastest migrating band) and unreacted starting porphyrin 1 (fourth band). Yields (crystallized from dichloromethane/hexane): 3, 35 $\operatorname{mg}(55 \%) ; 4,7 \mathrm{mg}(13 \%)$.

Selected data for 3: $\lambda_{\max }\left(\mathrm{CH}_{2} \mathrm{Cl}_{2}\right) / \mathrm{nm}$ (ع) 307 (32100), 364 (46500), 450 (113700), 616 (8900), 663 (12800), 718 (14900); HRMS (ESI) calc. for $\mathrm{C}_{56} \mathrm{H}_{46} \mathrm{~N}_{4} \mathrm{Br}\left([\mathrm{M}+1]^{+}\right): 853.2900$. Found: 853.2939. Anal. calc. for $\mathrm{C}_{56} \mathrm{H}_{45} \mathrm{~N}_{4} \mathrm{Br}\left(\mathrm{CH}_{2} \mathrm{Cl}_{2}\right)_{0.5} \mathrm{C}_{6} \mathrm{H}_{14}$ : C, 76.41; H, 5.93; N, 5.72. Found: C, 76.75; H, 5.82; N, 5.80. ${ }^{1} \mathrm{H}$ NMR $\left(500 \mathrm{MHz}, \mathrm{CDCl}_{3}, 298 \mathrm{~K}\right), \delta_{\mathrm{H}}=7.90\left(\mathrm{~d},{ }^{3} \mathrm{~J}=4.5\right.$ $\mathrm{Hz}, 1 \mathrm{H}) ; 7.79(\mathrm{~m}, 2 \mathrm{H}) ; 7.69\left(\mathrm{~d},{ }^{3} J=3.8 \mathrm{~Hz}, 1 \mathrm{H}\right) ; 7.68\left(\mathrm{~d},{ }^{3} J=3.8 \mathrm{~Hz}, 1 \mathrm{H}\right) ; 7.66(\mathrm{~m}, 2 \mathrm{H})$; $7.55\left(\mathrm{~d},{ }^{3} J=4.7 \mathrm{~Hz}, 1 \mathrm{H}\right) ; 7.48\left(\mathrm{~d},{ }^{3} J=4.7 \mathrm{~Hz}, 1 \mathrm{H}\right) ; 7.43(\mathrm{~m}, 2 \mathrm{H}) ; 7.37$ (m, 2H); $7.36\left(\mathrm{~d},{ }^{4} J\right.$ $=1.9 \mathrm{~Hz}, 1 \mathrm{H}) ; 7.35\left(\mathrm{~d},{ }^{3} \mathrm{~J}=4.7 \mathrm{~Hz}, 1 \mathrm{H}\right) ; 7.33(\mathrm{~m}, 2 \mathrm{H}) ; 7.19(\mathrm{~m}, 2 \mathrm{H}) ; 7.10\left(\mathrm{dd},{ }^{3} J=7.8 \mathrm{~Hz}\right.$, 
$\left.{ }^{4} J=0.7 \mathrm{~Hz}, 1 \mathrm{H}\right) ; 6.99\left(\mathrm{t},{ }^{3} J=7.7 \mathrm{~Hz}, 1 \mathrm{H}\right) ; 6.71\left(\mathrm{td},{ }^{3} J=7.7 \mathrm{~Hz},{ }^{4} J=0.7 \mathrm{~Hz}, 1 \mathrm{H}\right) ; 5.92(\mathrm{~d}$, $\left.{ }^{3} J=7.8 \mathrm{~Hz}, 1 \mathrm{H}\right) ; 5.20(\mathrm{~s}, 2 \mathrm{H}) ; 3.95(\mathrm{~b}, 1 \mathrm{H}) ; 3.93(\mathrm{~s}, 2 \mathrm{H}) ; 2.54(\mathrm{~s}, 3 \mathrm{H}) ; 2.53(\mathrm{~s}, 3 \mathrm{H}) ; 2.52(\mathrm{~s}$, 3H); $2.49(\mathrm{~s}, 3 \mathrm{H}) ; 1.73\left(\mathrm{~d},{ }^{4} \mathrm{~J}=1.9 \mathrm{~Hz}, 1 \mathrm{H}\right) .{ }^{13} \mathrm{C} \mathrm{NMR}\left(126 \mathrm{MHz}, \mathrm{CDCl}_{3}, 298 \mathrm{~K}\right), \delta_{\mathrm{C}}=$ $163.7,163.4,154.8,153.9,143.8,143.4,138.7,138.6,138.2,138.0,137.6,137.3,136.9$ (3CH), 136.8, 136.7, 136.7, 136.0, 135.0, 134.9, 134.9, 133.3, 133.2, 132.9, 131.1, 130.6, 130.5, $129.6,129.4,129.3,129.1,128.0,127.9,127.9,127.5,126.3,124.2,115.7,115.2,108.2(21-$ CH), 51.2, 30.5, 21.4, 21.4, 21.3.

Selected data for 4: $\lambda_{\max }\left(\mathrm{CH}_{2} \mathrm{Cl}_{2}\right) / \mathrm{nm}$ 307, 367, 450, 618, 665, 721; MS (ESI) calc. for $\mathrm{C}_{104} \mathrm{H}_{78} \mathrm{~N}_{8}:$ 1439.8. Found: $1444.2 .{ }^{1} \mathrm{H}$ NMR $\left(500 \mathrm{MHz}, \mathrm{CDCl}_{3}, 298 \mathrm{~K}\right), \delta_{\mathrm{H}}=7.95\left(\mathrm{~d},{ }^{3} J=4.3\right.$ $\mathrm{Hz}, 2 \mathrm{H}) ; 7.89(\mathrm{~m}, 4 \mathrm{H}) ; 7.70(\mathrm{~m}, 4 \mathrm{H}) ; 7.69\left(\mathrm{~d},{ }^{3} J=4.7 \mathrm{~Hz}, 2 \mathrm{H}\right) ; 7.68\left(\mathrm{~d},{ }^{3} J=4.7 \mathrm{~Hz}, 2 \mathrm{H}\right) ; 7.62$ $(\mathrm{m}, 4 \mathrm{H}) ; 7.52\left(\mathrm{~d},{ }^{3} J=4.3 \mathrm{~Hz}, 2 \mathrm{H}\right) ; 7.49(\mathrm{~m}, 4 \mathrm{H}) ; 7.37(\mathrm{~m}, 4 \mathrm{H}) ; 7.35\left(\mathrm{~d},{ }^{3} J=4.4 \mathrm{~Hz}, 2 \mathrm{H}\right) ; 7.33$ $\left(\mathrm{d},{ }^{4} J=1.7 \mathrm{~Hz}, 2 \mathrm{H}\right) ; 7.31(\mathrm{~m}, 4 \mathrm{H}) ; 7.29\left(\mathrm{~d},{ }^{3} \mathrm{~J}=4.4 \mathrm{~Hz}, 2 \mathrm{H}\right) ; 7.15(\mathrm{~m}, 4 \mathrm{H}) ; 6.90(\mathrm{~m}, 4 \mathrm{H}) ; 6.47$ $(\mathrm{m}, 2 \mathrm{H}) ; 5.70(\mathrm{~m}, 2 \mathrm{H}) ; 4.34(\mathrm{~s}, 4 \mathrm{H}) ; 3.86(\mathrm{~b}, 2 \mathrm{H}) ; 2.62(\mathrm{~s}, 6 \mathrm{H}) ; 2.55(\mathrm{~s}, 6 \mathrm{H}) ; 2.51(\mathrm{~s}, 6 \mathrm{H})$; $2.06(\mathrm{~s}, 6 \mathrm{H}) ; 1.77\left(\mathrm{~d},{ }^{4} J=1.7 \mathrm{~Hz}, 2 \mathrm{H}\right) .{ }^{13} \mathrm{C} \mathrm{NMR}\left(126 \mathrm{MHz}, \mathrm{CDCl}_{3}, 298 \mathrm{~K}\right), \delta_{\mathrm{C}}=163.7$; $163.5 ; 154.9 ; 153.9 ; 143.8 ; 143.5 ; 138.7 ; 138.6 ; 138.4 ; 137.7 ; 137.4 ; 137.3 ; 137.2$ (3-C); $136.8 ; 136.7 ; 135.4 ; 135.0 ; 134.8 ; 134.7 ; 134.0 ; 133.3 ; 133.2 ; 132.8 ; 130.8 ; 130.6 ; 129.3 ;$ $129.2 ; 127.9 ; 127.8 ; 127.5 ; 127.4 ; 124.3 ; 124.0 ; 115.8 ; 115.3 ; 109.1(21-\mathrm{C}) ; 51.3\left(\mathrm{CH}_{2}\right) ; 21.4$; $21.3 ; 20.8$.

Synthesis of 5. In a typical synthesis $50 \mathrm{mg}(0.075 \mathrm{mmole})$ of $\mathbf{1}$ was refluxed for $12 \mathrm{~h}$ in a dry toluene $(30 \mathrm{~mL})$ with $59 \mathrm{mg}(0.225 \mathrm{mmole})$ of 1,2-dibromomethylbenzene along with a suspension of $10 \mathrm{mg}$ of $\mathrm{K}_{2} \mathrm{CO}_{3}$ in the presence of air. Separation of the products was carried out by means of column chromatography with a neutral aluminum oxide column (activity $3^{\circ}$ ) as a stationary phase. Product $\mathbf{5}$ was eluted with a dichloromethane as a orange-red band and crystallized from dichloromethane/hexane. Yield: $26 \mathrm{mg}(45 \%)$. Selected data for 5: 
$\lambda_{\max }\left(\mathrm{CH}_{2} \mathrm{Cl}_{2}\right) / \mathrm{nm}(\varepsilon) 374$ (38000), 434 (sh), 460 (73600), 496 (53100), 524 (74000), 624

(10000), 677 (20000), 766 (sh); HRMS (ESI) calc. for $\mathrm{C}_{56} \mathrm{H}_{43} \mathrm{~N}_{4}\left([\mathrm{M}+1]^{+}\right)$: 771.3482. Found: 771.3497. Anal. calc. for $\mathrm{C}_{56} \mathrm{H}_{43} \mathrm{~N}_{4}$ : C, 87.27; H, 5.45; N, 7.27. Found: C, 86.90; H, 5.40; N, 7.50. ${ }^{1} \mathrm{H}$ NMR $\left(500 \mathrm{MHz}, \mathrm{CDCl}_{3}, 298 \mathrm{~K}\right), \delta_{\mathrm{H}}=8.78(\mathrm{~s}, 1 \mathrm{H}) ; 8.291\left(\mathrm{~d},{ }^{3} \mathrm{~J}=4.5 \mathrm{~Hz}, 1 \mathrm{H}\right) ; 8.287$ $\left(\mathrm{d},{ }^{3} J=4.7 \mathrm{~Hz}, 1 \mathrm{H}\right) ; 8.284\left(\mathrm{~d},{ }^{3} J=4.5 \mathrm{~Hz}, 1 \mathrm{H}\right) ; 8.276\left(\mathrm{~d},{ }^{3} J=4.5 \mathrm{~Hz}, 1 \mathrm{H}\right) ; 8.09(\mathrm{~m}, 2 \mathrm{H}) ; 8.05$ $(\mathrm{m}, 2 \mathrm{H}) ; 8.03\left(\mathrm{~d},{ }^{3} J=4.5 \mathrm{~Hz}, 1 \mathrm{H}\right) ; 8.01\left(\mathrm{~d},{ }^{3} J=4.5 \mathrm{~Hz}, 1 \mathrm{H}\right) ; 7.92(\mathrm{~m}, 4 \mathrm{H}) ; 7.61(\mathrm{~m}, 2 \mathrm{H}) ; 7.56$ (m, 2H); $7.44(\mathrm{~m}, 4 \mathrm{H}) ; 7.39(\mathrm{~m}, 1 \mathrm{H}) ; 7.33(\mathrm{~m}, 1 \mathrm{H}) ; 7.26(\mathrm{~m}, 1 \mathrm{H}) ; 7.25(\mathrm{~m}, 1 \mathrm{H}) ; 7.16(\mathrm{~s}, 1 \mathrm{H})$; $2.72(\mathrm{~s}, 3 \mathrm{H}) ; 2.70(\mathrm{~s}, 3 \mathrm{H}) ; 2.61(\mathrm{~s}, 3 \mathrm{H}) ; 0.66(\mathrm{~b}, 1 \mathrm{H}) ;-1.07(\mathrm{~s}, 1 \mathrm{H}) .{ }^{13} \mathrm{C}$ NMR $(126 \mathrm{MHz}$, $\left.\mathrm{CDCl}_{3}, 298 \mathrm{~K}\right), \delta_{\mathrm{C}}=158.8 ; 158.2 ; 153.6 ; 152.9 ; 141.8 ; 140.7 ; 140.0 ; 139.9 ; 139.8 ; 139.7 ;$ $138.3 ; 137.7 ; 137.3 ; 136.5 ; 136.5 ; 134.2 ; 134.1 ; 133.4 ; 133.2 ; 133.1 ; 130.9 ; 130.8 ; 130.2 ;$ $130.0 ; 129.4 ; 128.1 ; 127.9 ; 127.6 ; 127.5 ; 127.5 ; 127.5 ; 127.3 ; 124.8 ; 123.6 ; 119.3 ; 118.3 ;$ $117.5 ; 116.8 ; 115.7 ; 113.3(21-\mathrm{C}) ; 21.6 ; 21.5 ; 21.5$.

Synthesis of 6. A short column filled with the activated basic aluminum oxide (activity $1^{\circ}$ ) was charged with a benzene solution of $\mathbf{3}(20 \mathrm{mg}, 0.023 \mathrm{mmole})$ and the green band was developed with dichloromethane. The band was then eluted with a chloroform/ethanol mixture $(95 / 5 \mathrm{v} / \mathrm{v})$. Separation of the products was carried out by means of column chromatography with a neutral aluminum oxide column (activity $3^{\circ}$ ) as a stationary phase. Product 6 was eluted with a benzene/dichloromethane mixture (80/20 v/v) as a brownishgreen band and crystallized from dichloromethane/ethanol. Yield: $14 \mathrm{mg}$ (74\%). Selected data for 6: $\lambda_{\max }\left(\mathrm{CH}_{2} \mathrm{Cl}_{2}\right) / \mathrm{nm}$ (ع) 342 (20700), 400 (34500), 455 (118700), 551 (10400), 592 (5900), 644 (4900), 707 (8700); HRMS (ESI) calc. for $\mathrm{C}_{56} \mathrm{H}_{47} \mathrm{~N}_{4} \mathrm{O}\left([\mathrm{M}+1]^{+}\right)$: 791.3744. Found: 791.3775. Anal. calc. for $\mathrm{C}_{56} \mathrm{H}_{46} \mathrm{~N}_{4}$ : C, 85.06; H, 5.82; N, 7.09. Found: C, 85.36; H, 6.07; N, 7.01. ${ }^{1} \mathrm{H}$ NMR $\left(500 \mathrm{MHz}, \mathrm{CDCl}_{3}, 213 \mathrm{~K}\right), \delta_{\mathrm{H}}=8.48\left(\mathrm{~d},{ }^{3} J=4.6 \mathrm{~Hz}, 1 \mathrm{H}\right) ; 8.43(\mathrm{~s}, 2 \mathrm{H}) ; 8.42$ $\left(\mathrm{d},{ }^{3} J=5.0 \mathrm{~Hz}, 1 \mathrm{H}\right) ; 8.33(\mathrm{~m}, 1 \mathrm{H}) ; 8.31(\mathrm{~m}, 1 \mathrm{H}) ; 8.18\left(\mathrm{~d},{ }^{3} J=4.6 \mathrm{~Hz}, 1 \mathrm{H}\right) ; 8.10(\mathrm{~m}, 1 \mathrm{H})$; 
$8.08(\mathrm{~m}, 1 \mathrm{H}) ; 8.06(\mathrm{~s}, 1 \mathrm{H}) ; 8.05\left(\mathrm{~d},{ }^{3} J=5.0 \mathrm{~Hz}, 1 \mathrm{H}\right) ; 7.97(\mathrm{~m}, 1 \mathrm{H}) ; 7.90(\mathrm{~m}, 1 \mathrm{H}) ; 7.86(\mathrm{~m}$, $1 \mathrm{H}) ; 7.66(\mathrm{~s}, 2 \mathrm{H}) ; 7.56(\mathrm{~m}, 1 \mathrm{H}) ; 7.53(\mathrm{~m}, 2 \mathrm{H}) ; 7.50(\mathrm{~m}, 3 \mathrm{H}) ; 7.45(\mathrm{~m}, 1 \mathrm{H}) ; 6.79\left(\mathrm{t},{ }^{3} J=7.5\right.$ $\mathrm{Hz}, 1 \mathrm{H}) ; 6.56\left(\mathrm{t},{ }^{3} J=7.5 \mathrm{~Hz}, 1 \mathrm{H}\right) ; 6.48\left(\mathrm{~d},{ }^{3} J=7.6 \mathrm{~Hz}, 1 \mathrm{H}\right) ; 5.89\left(\mathrm{~d},{ }^{3} J=7.6 \mathrm{~Hz}, 1 \mathrm{H}\right) ; 4.79$ $\left(\mathrm{d},{ }^{2} J=16.7 \mathrm{~Hz}, 1 \mathrm{H}\right) ; 3.92\left(\mathrm{~d},{ }^{2} J=16.7 \mathrm{~Hz}, 1 \mathrm{H}\right) ; 3.34\left(\mathrm{~d},{ }^{2} J=15.5 \mathrm{~Hz}, 1 \mathrm{H}\right) ; 2.67(\mathrm{~s}, 3 \mathrm{H})$; $2.64(\mathrm{~s}, 3 \mathrm{H}) ; 2.61(\mathrm{~s}, 3 \mathrm{H}) ; 2.58(\mathrm{~s}, 3 \mathrm{H}) ; 1.90\left(\mathrm{~d},{ }^{2} \mathrm{~J}=15.5 \mathrm{~Hz}, 1 \mathrm{H}\right) ;-2.36(\mathrm{~b}, 1 \mathrm{H}) ;-2.71(\mathrm{~b}$, $1 \mathrm{H}) ;-4.62(\mathrm{~s}, 1 \mathrm{H}) .{ }^{13} \mathrm{C} \mathrm{NMR}\left(126 \mathrm{MHz}, \mathrm{CDCl}_{3}, 298 \mathrm{~K}\right), \delta_{\mathrm{C}}=153.6 ; 151.9 ; 148.5 ; 140.3$; $140.0 ; 139.5 ; 139.3 ; 137.9 ; 137.8 ; 137.6 ; 137.2 ; 137.2 ; 137.1 ; 137.0 ; 137.0 ; 136.6 ; 135.6$; $134.9 ; 134.8 ; 134.4 ; 134.1 ; 133.7 ; 133.6 ; 133.4 ; 133.3 ; 132.0 ; 131.1 ; 129.3 ; 128.8 ; 128.5 ;$ $127.9 ; 127.9 ; 127.6 ; 127.5 ; 127.4 ; 126.2 ; 125.9 ; 125.6 ; 125.4 ; 124.1 ; 122.4 ; 121.0 ; 118.6$; 113.9 (21-C); 104.9; 99.2 (3-C); 65.6; 48.4; 21.6; 21.5; 21.5; 21.5.

Insertion of the silver(III) ion. To the solution of $6(10 \mathrm{mg})$ in THF $(10 \mathrm{ml})$ an excess of silver(I) tetrafluoroborate $(20 \mathrm{mg}$ ) was added and the mixture was stirred at room temperature for one hour at room temperature. The solvent was then removed and the solid residue was extracted with three portions of benzene $(5 \mathrm{ml}$ each). The extracts were joined, filtered, and the benzene solvent was then evaporated. The product 7 was characterized only in solution due to its further alteration during the purification attempt. Selected data for 7: $\lambda_{\max }\left(\mathrm{CH}_{2} \mathrm{Cl}_{2}\right) / \mathrm{nm} \quad 254$ (sh), 324, 375, 454, 529, 574, 640, 693; MS (ESI) calc. for $\mathrm{C}_{56} \mathrm{H}_{44} \mathrm{~N}_{4} \mathrm{OAg}\left([\mathrm{M}]^{+}\right)$: 896.9. Found: 896.2. ${ }^{1} \mathrm{H}$ NMR (500 MHz, $\left.\mathrm{CDCl}_{3}, 298 \mathrm{~K}\right), \delta_{\mathrm{H}}=9.27$ $(\mathrm{s}, 1 \mathrm{H}) ; 8.79\left(\mathrm{~d},{ }^{3} \mathrm{~J}=4.5 \mathrm{~Hz}, 1 \mathrm{H}\right) ; 8.49\left(\mathrm{~d},{ }^{3} \mathrm{~J}=4.5 \mathrm{~Hz}, 1 \mathrm{H}\right) ; 8.44\left(\mathrm{~d},{ }^{3} J=5.0 \mathrm{~Hz}, 1 \mathrm{H}\right) ; 8.41$ $\left(\mathrm{d},{ }^{3} J=5.0 \mathrm{~Hz}, 1 \mathrm{H}\right) ; 8.36\left(\mathrm{~d},{ }^{3} J=4.8 \mathrm{~Hz}, 1 \mathrm{H}\right) ; 8.27\left(\mathrm{~d},{ }^{3} J=4.8 \mathrm{~Hz}, 1 \mathrm{H}\right) ; 7.93(\mathrm{~m}, 2 \mathrm{H}) ; 7.88$ $(\mathrm{m}, 2 \mathrm{H}) ; 7.85(\mathrm{~m}, 2 \mathrm{H}) ; 7.60(\mathrm{~m}, 2 \mathrm{H}) ; 7.54(\mathrm{~m}, 4 \mathrm{H}) ; 7.51(\mathrm{~m}, 2 \mathrm{H}) ; 7.35(\mathrm{~m}, 2 \mathrm{H}) ; 7.18(\mathrm{~m}$, $1 \mathrm{H}) ; 6.87(\mathrm{~m}, 1 \mathrm{H}) ; 6.36\left(\mathrm{~d},{ }^{3} \mathrm{~J}=8.3 \mathrm{~Hz}, 1 \mathrm{H}\right) ; 5.97(\mathrm{~s}, 2 \mathrm{H}) ; 4.44(\mathrm{~s}, 2 \mathrm{H}) ; 3.43(\mathrm{~b}, 1 \mathrm{H}) ; 2.66(\mathrm{~s}$, $3 \mathrm{H}) ; 2.64$ (s, 3H); 2.60 (s, 3H); 2.57 (s, 3H). 
Conversion of 7 into 8. To the chloroform solution containing 7 a drop of water and $2 \mathrm{mg}$ of potassium carbonate was added and the mixture was vigorously stirred for 5 minutes. The organic layer was then separated, washed with small portion of water, and solvent was evaporated. The product 8 was characterized only in solution due to its oxidative transformation into $\mathbf{1 0}$ during the purification attempt. Selected data for 8: $\lambda_{\max }\left(\mathrm{CH}_{2} \mathrm{Cl}_{2}\right) / \mathrm{nm}$ 269, 386, 450, 529, 566, 640,; MS (ESI) calc. for $\mathrm{C}_{56} \mathrm{H}_{44} \mathrm{~N}_{4} \mathrm{OAg}\left([\mathrm{M}+1]^{+}\right)$: 896.9. Found: 896.2. ${ }^{1} \mathrm{H} \mathrm{NMR}\left(500 \mathrm{MHz}, \mathrm{CDCl}_{3}, 298 \mathrm{~K}\right), \delta_{\mathrm{H}}=8.56\left(\mathrm{dd},{ }^{3} J_{\mathrm{HH}}=4.8 \mathrm{~Hz} ;{ }^{4} J_{\mathrm{AgH}}=0.9 \mathrm{~Hz}\right.$, $1 \mathrm{H}) ; 8.51\left(\mathrm{dd},{ }^{3} J_{\mathrm{HH}}=4.8 \mathrm{~Hz} ;{ }^{4} J_{\mathrm{AgH}}=1.4 \mathrm{~Hz}, 1 \mathrm{H}\right) ; 8.46\left(\mathrm{dd},{ }^{3} J_{\mathrm{HH}}=4.6 \mathrm{~Hz} ; 1 \mathrm{H}\right) ; 8.42\left(\mathrm{dd},{ }^{3} J_{\mathrm{HH}}\right.$ $=4.6 \mathrm{~Hz} ; 1 \mathrm{H}) ; 8.40\left(\mathrm{dd},{ }^{3} J_{\mathrm{HH}}=4.8 \mathrm{~Hz} ;{ }^{4} J_{\mathrm{AgH}}=0.9 \mathrm{~Hz}, 1 \mathrm{H}\right) ; 8.23\left(\mathrm{dd},{ }^{3} J_{\mathrm{HH}}=4.8 \mathrm{~Hz} ;{ }^{4} J_{\mathrm{AgH}}=\right.$ $1.4 \mathrm{~Hz}, 1 \mathrm{H}) ; 7.97(\mathrm{~m}, 2 \mathrm{H}) ; 7.93(\mathrm{~m}, 2 \mathrm{H}) ; 7.85(\mathrm{~m}, 1 \mathrm{H}) ; 7.79(\mathrm{~m}, 1 \mathrm{H}) ; 7.68(\mathrm{~s}, 1 \mathrm{H}) ; 7.63(\mathrm{~m}$, $2 \mathrm{H}) ; 7.53(\mathrm{~m}, 1 \mathrm{H}) ; 7.50(\mathrm{~m}, 2 \mathrm{H}) ; 7.48(\mathrm{~m}, 2 \mathrm{H}) ; 7.45(\mathrm{~m}, 2 \mathrm{H}) ; 7.20(\mathrm{~m}, 1 \mathrm{H}) ; 7.00\left(\mathrm{t},{ }^{3} J_{\mathrm{HH}}=7.4\right.$ $\mathrm{Hz}, 1 \mathrm{H}) ; 6.85\left(\mathrm{t},{ }^{3} J_{\mathrm{HH}}=7.3 \mathrm{~Hz}, 1 \mathrm{H}\right) ; 6.66\left(\mathrm{~d},{ }^{3} J_{\mathrm{HH}}=7.3 \mathrm{~Hz}, 1 \mathrm{H}\right) ; 6.44\left(\mathrm{~d},{ }^{3} J_{\mathrm{HH}}=7.3 \mathrm{~Hz}, 1 \mathrm{H}\right)$; $4.95\left(\mathrm{~d},{ }^{2} J_{\mathrm{HH}}=17.2 \mathrm{~Hz}, 1 \mathrm{H}\right) ; 4.34\left(\mathrm{~d},{ }^{2} J_{\mathrm{HH}}=17.2 \mathrm{~Hz}, 1 \mathrm{H}\right) ; 3.99\left(\mathrm{~d},{ }^{2} J_{\mathrm{HH}}=15.2 \mathrm{~Hz}, 1 \mathrm{H}\right) ; 3.86$ $\left(\mathrm{d},{ }^{2} J_{\mathrm{HH}}=15.2 \mathrm{~Hz}, 1 \mathrm{H}\right) ; 2.66(\mathrm{~s}, 3 \mathrm{H}) ; 2.65(\mathrm{~s}, 3 \mathrm{H}) ; 2.62(\mathrm{~s}, 3 \mathrm{H}) ; 2.60(\mathrm{~s}, 3 \mathrm{H})$.

Syntheses of 9 and 10. To the solution of $6(10 \mathrm{mg})$ in dichloromethane $(10 \mathrm{ml})$ a solution of 20 of silver(I) acetate in acetonitrile was added and the mixture was stirred for $3 \mathrm{~h}$. The solvents was then removed and the solid residue was extracted with dichloromethane. The extract was passed down a silicagel column. First bright-red band contained 9, while the second brownish-red migrating with chloroform, consisted of $\mathbf{1 0}$. Both products were crystallized from dichloromethane/hexane. Yields: 9, $6 \mathrm{mg}$ (50\%); 10, $5 \mathrm{mg}$ (40\%). Selected data for 9: $\lambda_{\max }\left(\mathrm{CH}_{2} \mathrm{Cl}_{2}\right) / \mathrm{nm}(\varepsilon) 283$ (26100), 360 (21000), 372 (20500), 450 (137800), 529 (9754), 574 (11600), 613 (4000); MS (ESI) calc. for $\mathrm{C}_{58} \mathrm{H}_{45} \mathrm{~N}_{4} \mathrm{O}_{3} \mathrm{Ag}\left([\mathrm{M}]^{+}\right)$: 953.9. Found: 953.5. ${ }^{1} \mathrm{H} \mathrm{NMR}\left(500 \mathrm{MHz}, \mathrm{CDCl}_{3}, 298 \mathrm{~K}\right), \delta_{\mathrm{H}}=8.66\left(\mathrm{dd},{ }^{3} J_{\mathrm{HH}}=5.0 \mathrm{~Hz},{ }^{4} J_{\mathrm{AgH}}=1.1 \mathrm{~Hz}, 1 \mathrm{H}\right)$; $8.62\left(\mathrm{dd},{ }^{3} J_{\mathrm{HH}}=4.8 \mathrm{~Hz},{ }^{4} J_{\mathrm{AgH}}=1.4 \mathrm{~Hz}, 1 \mathrm{H}\right) ; 8.60\left(\mathrm{~d},{ }^{3} J_{\mathrm{HH}}=4.8 \mathrm{~Hz}, 1 \mathrm{H}\right) ; 8.57\left(\mathrm{~d},{ }^{3} J_{\mathrm{HH}}=4.8\right.$ 
$\mathrm{Hz}, 1 \mathrm{H}) ; 8.52\left(\mathrm{dd},{ }^{3} J_{\mathrm{HH}}=4.8 \mathrm{~Hz},{ }^{4} J_{\mathrm{AgH}}=1.0 \mathrm{~Hz}, 1 \mathrm{H}\right) ; 8.23\left(\mathrm{dd},{ }^{3} J_{\mathrm{HH}}=5.0 \mathrm{~Hz},{ }^{4} J_{\mathrm{AgH}}=1.6 \mathrm{~Hz}\right.$, 1H); $7.93(\mathrm{~m}, 2 \mathrm{H}) ; 7.89(\mathrm{~m}, 2 \mathrm{H}) ; 7.76(\mathrm{~m}, 2 \mathrm{H}) ; 7.49(\mathrm{~m}, 2 \mathrm{H}) ; 7.46(\mathrm{~m}, 2 \mathrm{H}) ; 7.43(\mathrm{~m}, 2 \mathrm{H})$; $7.37(\mathrm{~m}, 2 \mathrm{H}) ; 7.19\left(\mathrm{~d},{ }^{3} J_{\mathrm{HH}}=7.3 \mathrm{~Hz}, 1 \mathrm{H}\right) ; 7.12(\mathrm{~m}, 2 \mathrm{H}) ; 6.99(\mathrm{~m}, 1 \mathrm{H}) ; 6.61(\mathrm{~m}, 2 \mathrm{H}) ; 5.25(\mathrm{~s}$, $2 \mathrm{H}) ; 4.64(\mathrm{~s}, 2 \mathrm{H}) ; 2.61(\mathrm{~s}, 3 \mathrm{H}) ; 2.58(\mathrm{~s}, 3 \mathrm{H}) ; 2.55(\mathrm{~s}, 3 \mathrm{H}) ; 2.49(\mathrm{~s}, 3 \mathrm{H}) ; 2.03(\mathrm{~s}, 3 \mathrm{H}) .{ }^{13} \mathrm{C}$ NMR $\left(126 \mathrm{MHz}, \mathrm{CDCl}_{3}, 298 \mathrm{~K}\right), \delta_{\mathrm{C}}=170.8 ; 169.8 ; 140.1 ; 139.4 ; 138.6 ; 138.6 ; 138.5 ; 138.4$; $138.2 ; 137.7 ; 137.6 ; 137.0 ; 136.1 ; 135.8 ; 133.8 ; 133.6 ; 132.6 ; 132.4 ; 131.8 ; 130.0 ; 129.9 ;$ $128.8 ; 128.6 ; 128.6 ; 128.5 ; 128.3 ; 128.2 ; 128.1 ; 128.1 ; 127.8 ; 127.7 ; 127.4 ; 126.4 ; 126.4 ;$ $126.1 ; 125.2 ; 124.5 ; 122.4 ; 120.9 ; 116.0 ; 114.4 ; 113.6 ; 63.8 ; 43.6 ; 21.6 ; 21.5 ; 21.4 ; 21.0$.

Selected data for 10: $\lambda_{\max }\left(\mathrm{CH}_{2} \mathrm{Cl}_{2}\right) / \mathrm{nm}$ 283, 360, 372, 450, 529, 574, 613; MS (ESI) calc. for $\mathrm{C}_{56} \mathrm{H}_{44} \mathrm{~N}_{4} \mathrm{O}_{2} \mathrm{Ag}\left([\mathrm{M}+1]^{+}\right)$: 912.9. Found: 912.7. ${ }^{1} \mathrm{H} \mathrm{NMR}\left(500 \mathrm{MHz}, \mathrm{CDCl}_{3}, 298 \mathrm{~K}\right), \delta_{\mathrm{H}}=$ $8.74\left(\mathrm{dd},{ }^{3} J_{\mathrm{HH}}=4.8 \mathrm{~Hz},{ }^{4} J_{\mathrm{AgH}}=1.5 \mathrm{~Hz}, 1 \mathrm{H}\right) ; 8.71\left(\mathrm{dd},{ }^{3} J_{\mathrm{HH}}=4.8 \mathrm{~Hz},{ }^{4} J_{\mathrm{AgH}}=0.9 \mathrm{~Hz}, 1 \mathrm{H}\right)$; $8.65\left(\mathrm{~d},{ }^{3} J_{\mathrm{HH}}=4.8 \mathrm{~Hz}, 1 \mathrm{H}\right) ; 8.62\left(\mathrm{~d},{ }^{3} J_{\mathrm{HH}}=4.8 \mathrm{~Hz}, 1 \mathrm{H}\right) ; 8.59\left(\mathrm{dd},{ }^{3} J_{\mathrm{HH}}=4.8 \mathrm{~Hz},{ }^{4} J_{\mathrm{AgH}}=0.9\right.$ $\mathrm{Hz}, 1 \mathrm{H}) ; 8.27\left(\mathrm{dd},{ }^{3} J_{\mathrm{HH}}=4.8 \mathrm{~Hz},{ }^{4} J_{\mathrm{AgH}}=1.4 \mathrm{~Hz}, 1 \mathrm{H}\right) ; 7.99(\mathrm{~m}, 2 \mathrm{H}) ; 7.96(\mathrm{~m}, 2 \mathrm{H}) ; 7.84(\mathrm{~m}$, 2H); $7.53(\mathrm{~m}, 2 \mathrm{H}) ; 7.50(\mathrm{~m}, 2 \mathrm{H}) ; 7.49(\mathrm{~m}, 2 \mathrm{H}) ; 7.46(\mathrm{~m}, 2 \mathrm{H}) ; 7.16(\mathrm{~m}, 2 \mathrm{H}) ; 7.24(\mathrm{~m}, 1 \mathrm{H})$; $7.00(\mathrm{~m}, 1 \mathrm{H}) ; 6.59(\mathrm{~m}, 2 \mathrm{H}) ; 5.40(\mathrm{~s}, 2 \mathrm{H}) ; 4.33\left(\mathrm{~d},{ }^{3} J_{\mathrm{HH}}=6.0 \mathrm{~Hz}, 2 \mathrm{H}\right) ; 2.67(\mathrm{~s}, 3 \mathrm{H}) ; 2.65(\mathrm{~s}$, $3 \mathrm{H}) ; 2.59(\mathrm{~s}, 3 \mathrm{H}) ; 2.55(\mathrm{~s}, 3 \mathrm{H}) ; 1.38\left(\mathrm{t},{ }^{3} \mathrm{~J}_{\mathrm{HH}}=6.0 \mathrm{~Hz}, 1 \mathrm{H}\right) .{ }^{13} \mathrm{C} \mathrm{NMR}\left(126 \mathrm{MHz}, \mathrm{CDCl}_{3}, 298\right.$ $\mathrm{K}), \delta_{\mathrm{C}}=169.8 ; 140.0 ; 139.4 ; 138.6 ; 138.6 ; 138.0 ; 137.7 ; 137.5 ; 137.2 ; 136.9 ; 136.3 ; 135.8 ;$ $133.8133 .6 ; 132.7 ; 131.8 ; 130.0 ; 129.9 ; 128.6 ; 128.5 ; 128.3 ; 128.2 ; 127.8 ; 127.7 ; 127.6$; $127.5 ; 127.3 ; 126.4 ; 126.3 ; 125.2 ; 124.7 ; 122.4 ; 120.9 ; 119.2 ; 116.5 ; 115.8 ; 62.8 ; 43.5 ; 21.6$; $21.5 ; 21.4$.

Synthesis of 11. To the solution of $5(15 \mathrm{mg})$ in THF $(10 \mathrm{ml})$ an excess of silver(I) tetrafluoroborate $(20 \mathrm{mg})$ was added and the mixture was stirred at room temperature for 30 minutes at room temperature. The solution turned olive green. The solvent was then removed and the solid residue was extracted with three portions of benzene ( $5 \mathrm{ml}$ each). The extracts 
were joined, filtered, and the benzene solvent was then evaporated. Crystallization from a dichloromethane/hexane mixture yielded $12 \mathrm{mg}$ of 11 (70\%). Selected data for 11: $\lambda_{\max }\left(\mathrm{CH}_{2} \mathrm{Cl}_{2}\right) / \mathrm{nm}$ (ع) 224 (82800), 268 (75600) 294 (sh), 404 (35200), 433 (55900); 461 (sh), 481 (119900), 538 (12451), 590 (sh), 614 (38800); MS (ESI) calc. for $\left[\mathrm{C}_{56} \mathrm{H}_{40} \mathrm{~N}_{4} \mathrm{Ag}\right]^{+}: 876.8$. Found: 877.0. Anal. calc. for $\mathrm{C}_{56} \mathrm{H}_{40} \mathrm{~N}_{4} \mathrm{AgBF}_{4} \mathrm{CH}_{2} \mathrm{Cl}_{2}$ : C, 65.20; $\mathrm{H}, 4.00 ; \mathrm{N}, 5.34$. Found: $\mathrm{C}$, 65.49; H, 3.92; N, 5.23. ${ }^{1} \mathrm{H}$ NMR $\left(500 \mathrm{MHz}, \mathrm{CDCl}_{3}, 298 \mathrm{~K}\right), \delta_{\mathrm{H}}=9.47(\mathrm{~s}, 1 \mathrm{H}) ; 8.82\left(\mathrm{dd},{ }^{3} J_{\mathrm{HH}}\right.$ $\left.=4.8 \mathrm{~Hz},{ }^{4} J_{\mathrm{AgH}}=1.3 \mathrm{~Hz}, 1 \mathrm{H}\right) ; 8.77\left(\mathrm{dd},{ }^{3} J_{\mathrm{HH}}=5.0 \mathrm{~Hz},{ }^{4} J_{\mathrm{AgH}}=1.1 \mathrm{~Hz}, 1 \mathrm{H}\right) ; 8.76\left(\mathrm{dd},{ }^{3} J_{\mathrm{HH}}=\right.$ $\left.4.8 \mathrm{~Hz},{ }^{4} J_{\mathrm{AgH}}=1.1 \mathrm{~Hz}, 1 \mathrm{H}\right) ; 8.75\left(\mathrm{dd},{ }^{3} J_{\mathrm{HH}}=4.8 \mathrm{~Hz},{ }^{4} J_{\mathrm{AgH}}=1.3 \mathrm{~Hz}, 1 \mathrm{H}\right) ; 8.72\left(\mathrm{~d},{ }^{3} J_{\mathrm{HH}}=4.4\right.$ $\mathrm{Hz}, 1 \mathrm{H}) ; 8.71\left(\mathrm{~d},{ }^{3} J_{\mathrm{HH}}=4.4 \mathrm{~Hz}, 1 \mathrm{H}\right) ; 8.02(\mathrm{~m}, 2 \mathrm{H}) ; 7.99(\mathrm{~m}, 2 \mathrm{H}) ; 7.98(\mathrm{~m}, 2 \mathrm{H}) ; 7.97(\mathrm{~m}, 2 \mathrm{H})$; $7.93(\mathrm{~m}, 1 \mathrm{H}) ; 7.82(\mathrm{~m}, 2 \mathrm{H}) ; 7.79(\mathrm{~m}, 1 \mathrm{H}) ; 7.73(\mathrm{~m}, 2 \mathrm{H}) ; 7.71(\mathrm{~m}, 1 \mathrm{H}) ; 7.68(\mathrm{~m}, 1 \mathrm{H}) ; 7.64(\mathrm{~s}$, 1H); $7.57(\mathrm{~m}, 4 \mathrm{H}) ; 2.81(\mathrm{~s}, 6 \mathrm{H}) ; 2.69(\mathrm{~s}, 6 \mathrm{H}) .{ }^{13} \mathrm{C} \mathrm{NMR}\left(126 \mathrm{MHz}, \mathrm{CDCl}_{3}, 298 \mathrm{~K}\right), \delta_{\mathrm{C}}=$ $144.8 ; 141.1 ; 140.9 ; 140.8 ; 140.6 ; 140.5 ; 140.1 ; 139.9 ; 138.7 ; 138.5 ; 137.4 ; 136.6 ; 135.9 ;$ $135.8 ; 134.2 ; 133.9 ; 133.8 ; 132.1 ; 131.7 ; 131.5 ; 131.4 ; 131.3 ; 130.7 ; 130.4 ; 130.3 ; 130.1 ;$ $129.9 ; 129.8 ; 129.6 ; 128.2 ; 128.1 ; 125.7 ; 125.0 ; 123.4 ; 123.1 ; 123.0 ; 121.3 ; 117.9 ; 117.9$; $115.0 ; 21.7 ; 21.5$. 


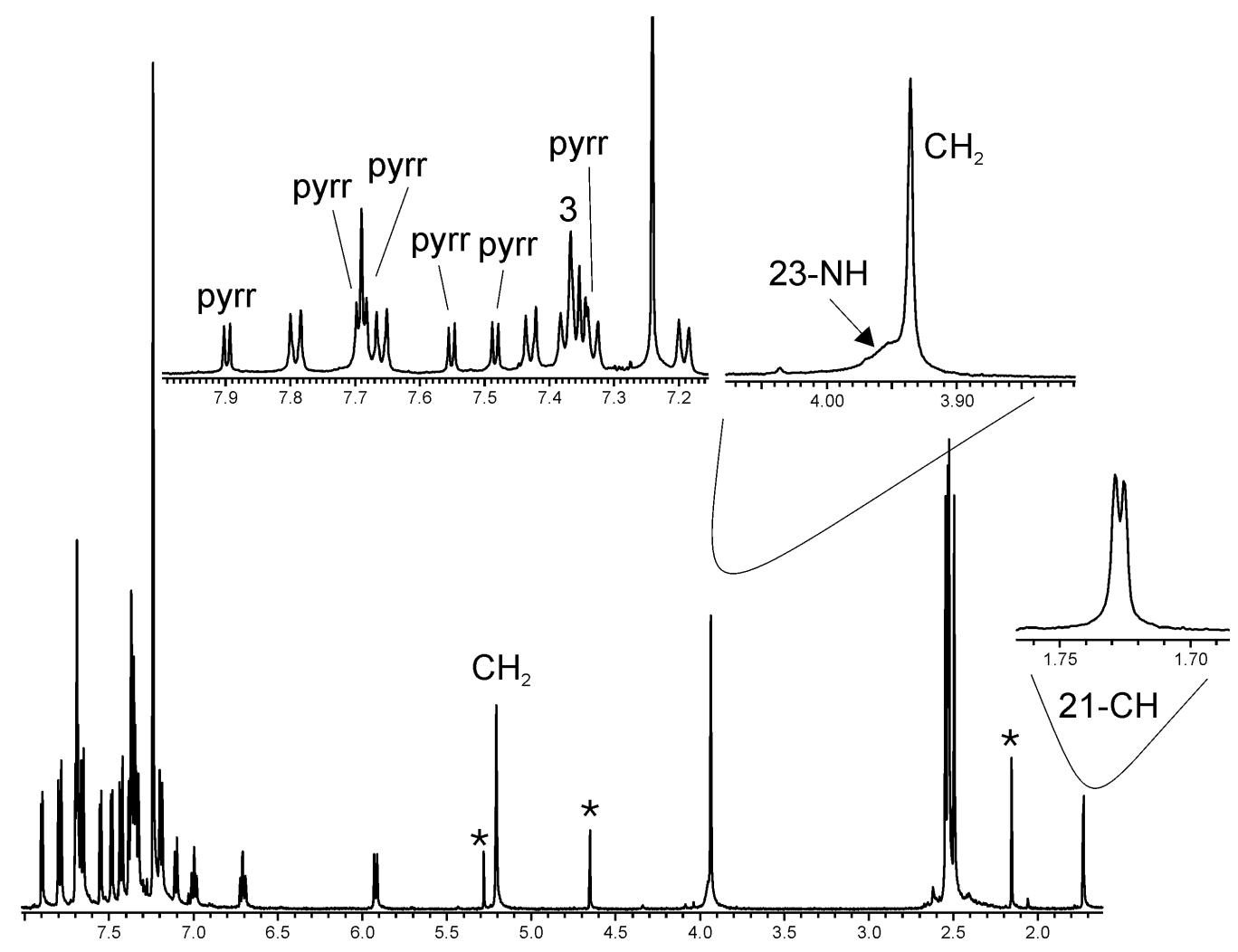

Fig. S1. ${ }^{1} \mathrm{H}$ NMR spectrum of 3, $298 \mathrm{~K}, \mathrm{CDCl}_{3}$. Assignments: pyrr, $\beta$-pyrrole, * - impurities.

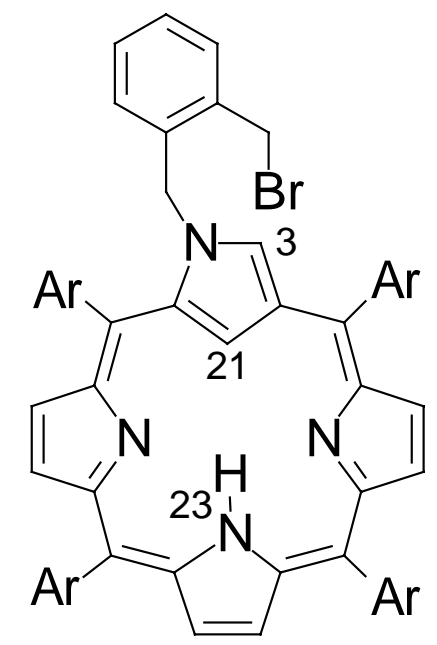

3

$$
\operatorname{Ar}=p-\text { Tol }
$$



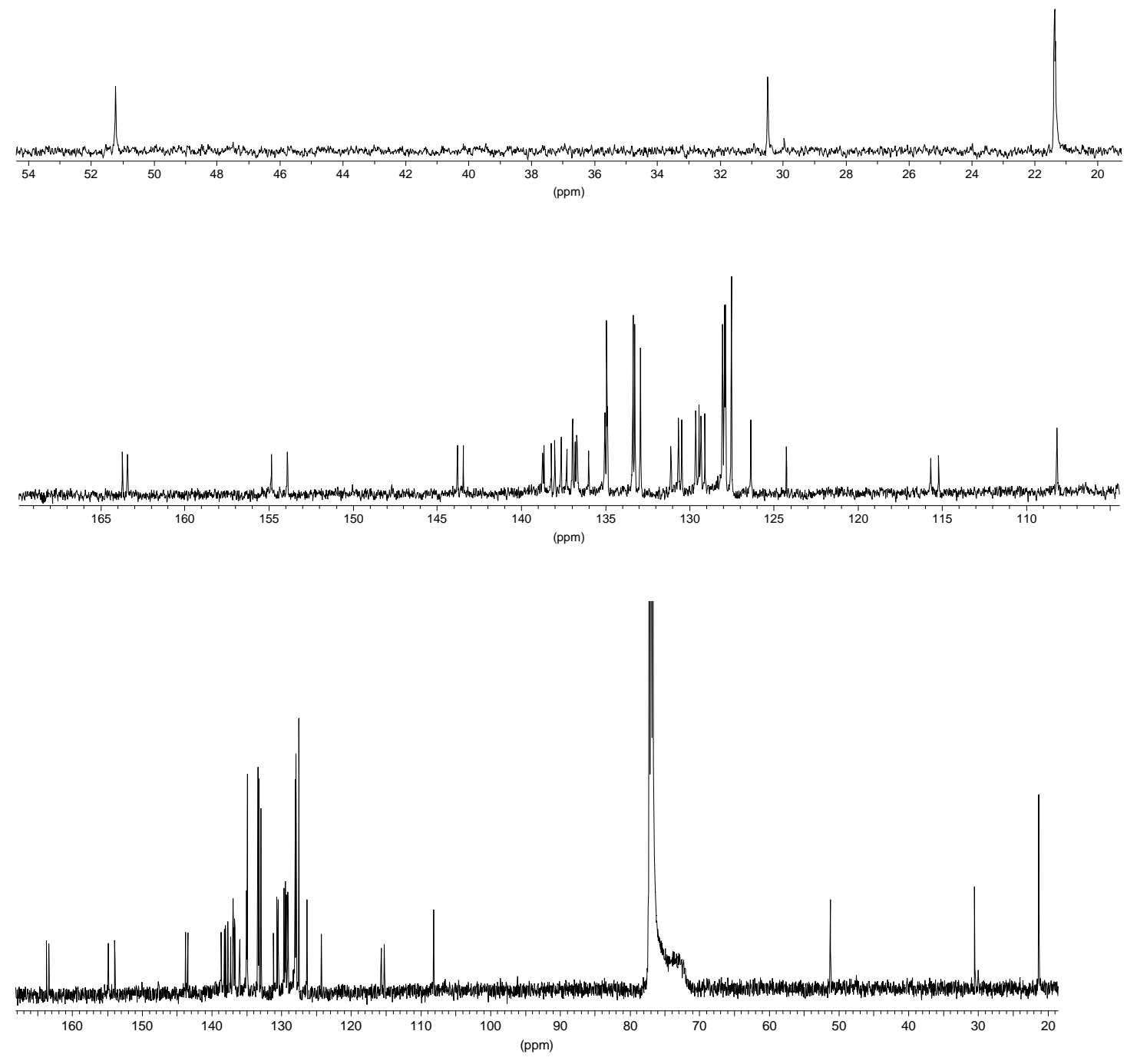

Fig. S2. ${ }^{13} \mathrm{C}$ NMR spectrum of $3,298 \mathrm{~K}, \mathrm{CDCl}_{3}$. 

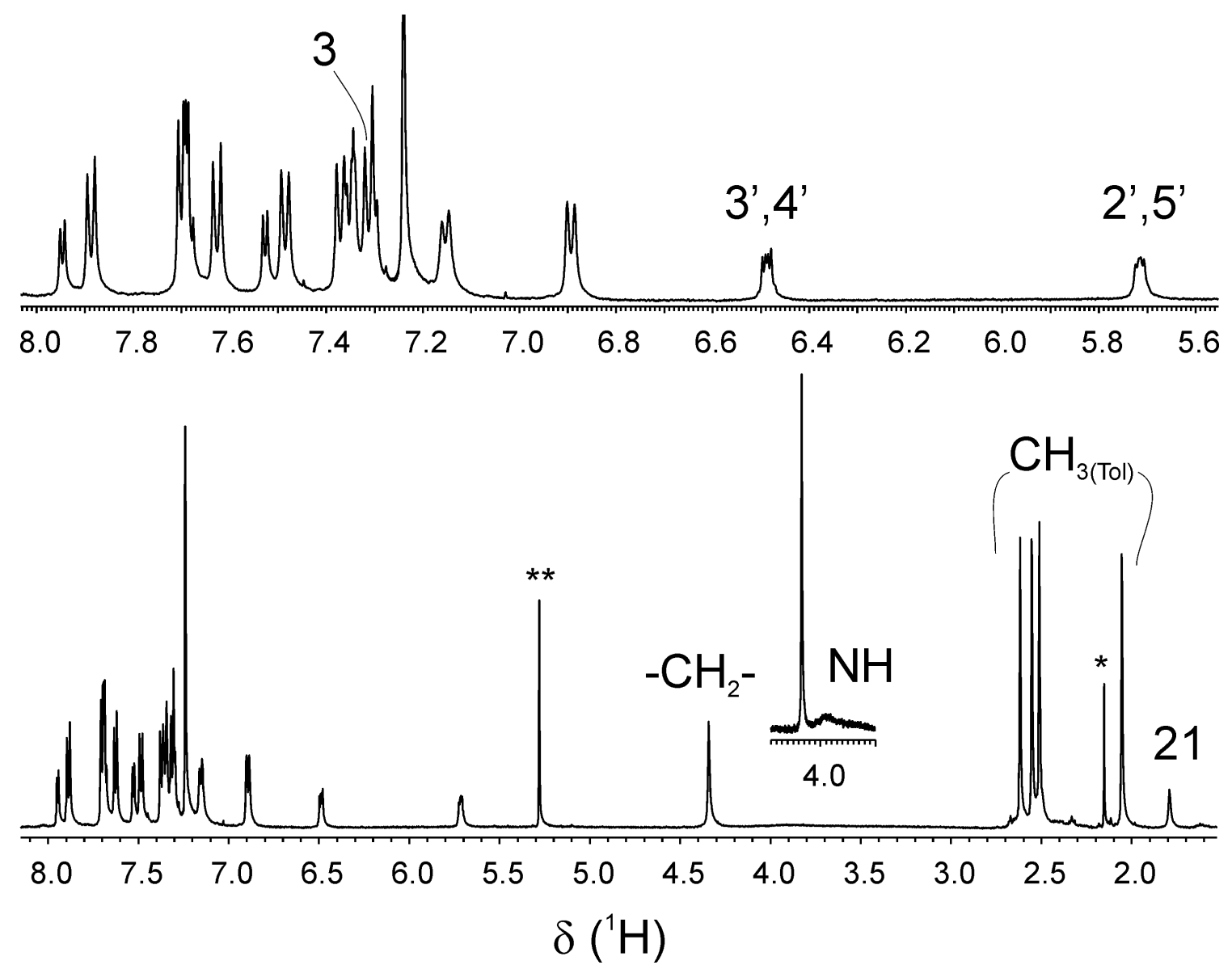

Fig. S3. ${ }^{1} \mathrm{H}$ NMR spectrum of dimer $4, \mathrm{CDCl}_{3}, 298 \mathrm{~K}$. *, **- impurities.

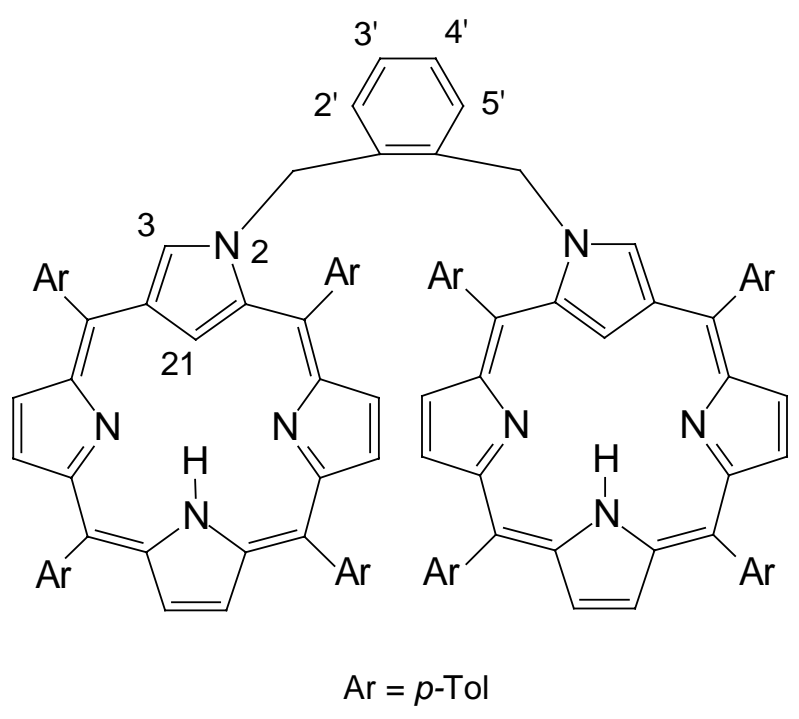




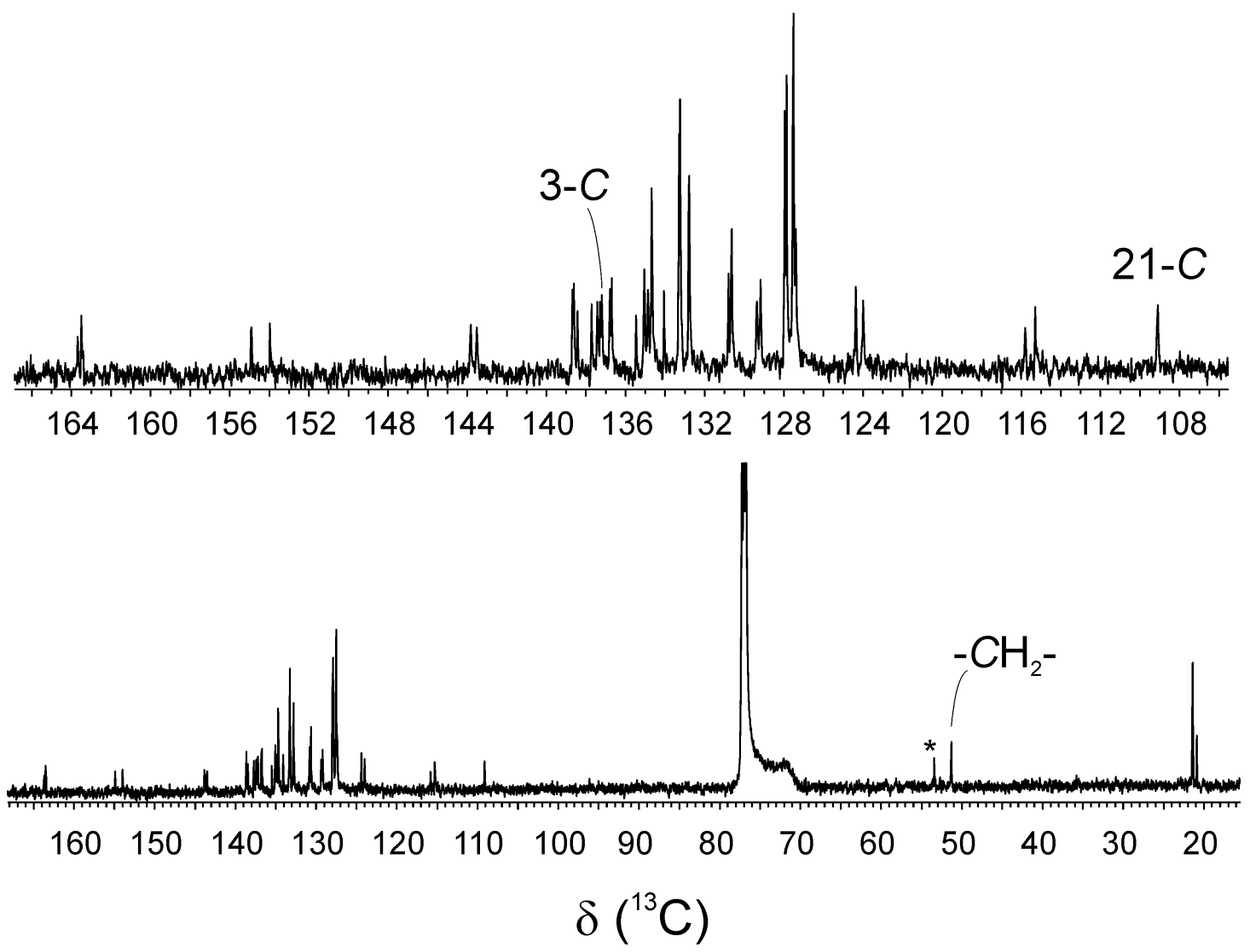

Fig. S4. ${ }^{13} \mathrm{C}$ NMR spectrum of dimer 4, $\mathrm{CDCl}_{3}, 298 \mathrm{~K} . *$ - dichloromethane from crystallization.

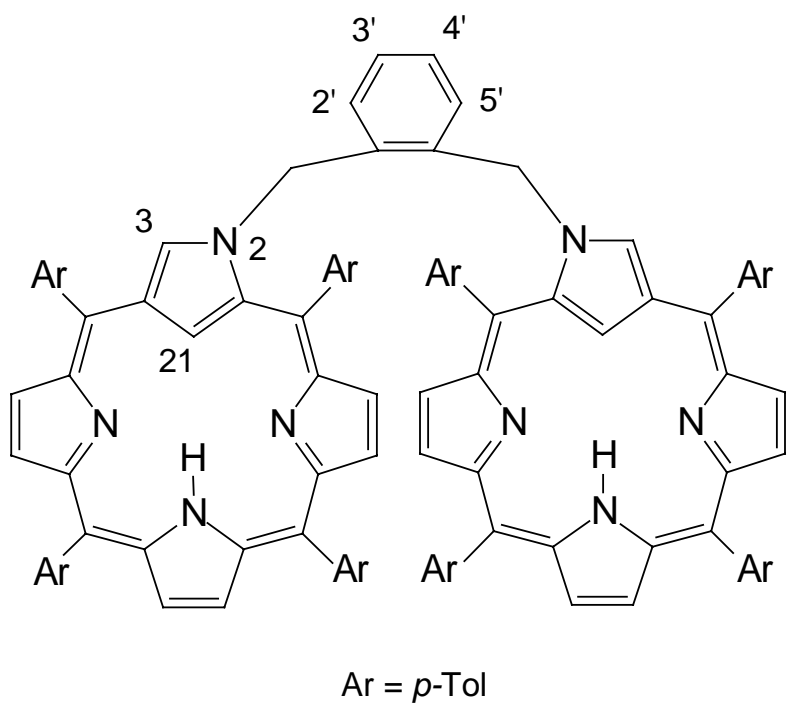



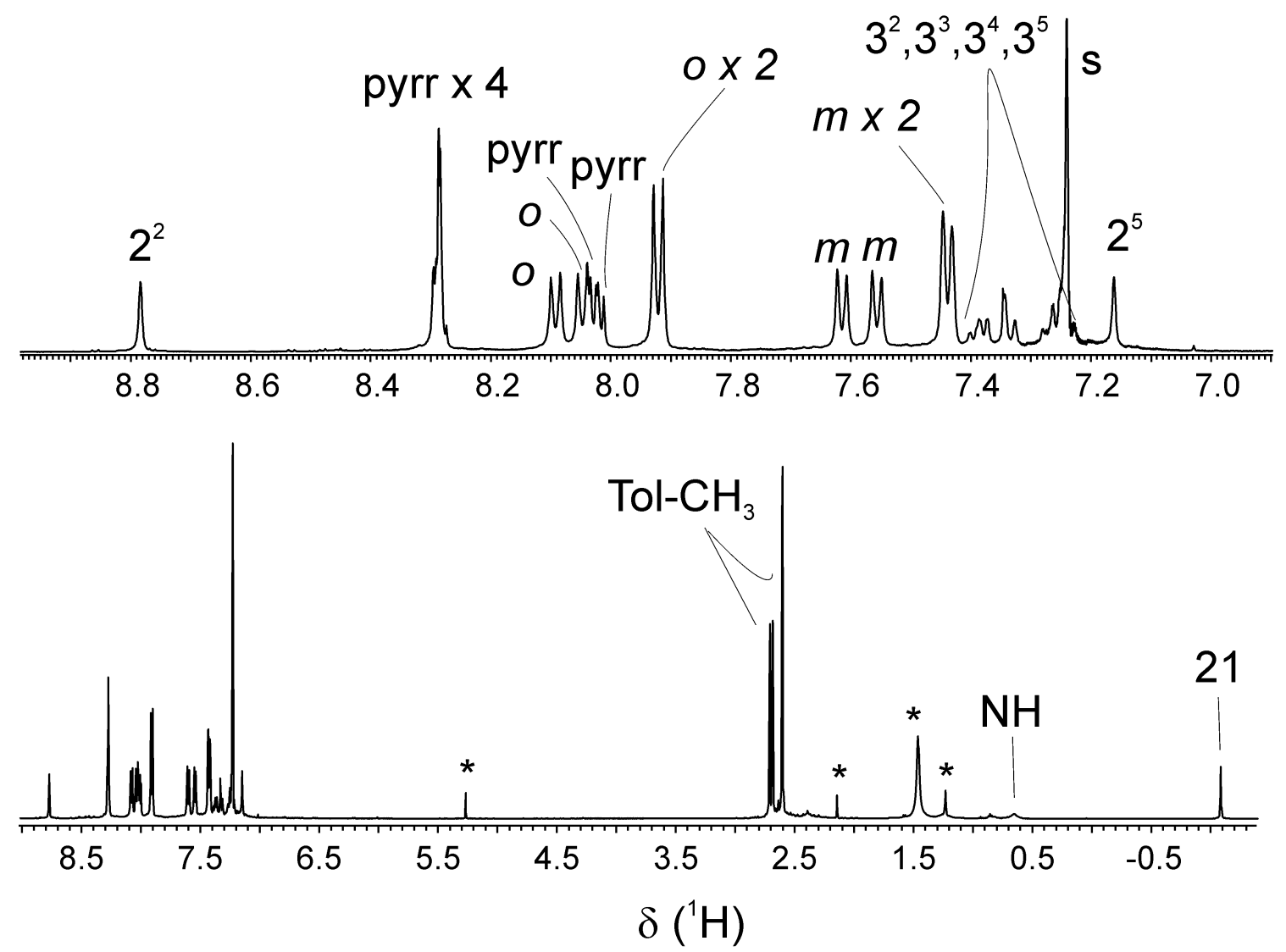

Fig. S5. ${ }^{1} \mathrm{H}$ NMR spectrum of $\mathbf{5}, \mathrm{CDCl}_{3}, 298 \mathrm{~K}$. Assignments: pyrr, $\beta$-pyrrole, $o, m$ - ortho and meta protons of meso-tolyl substituents, respectively, * - impurities.

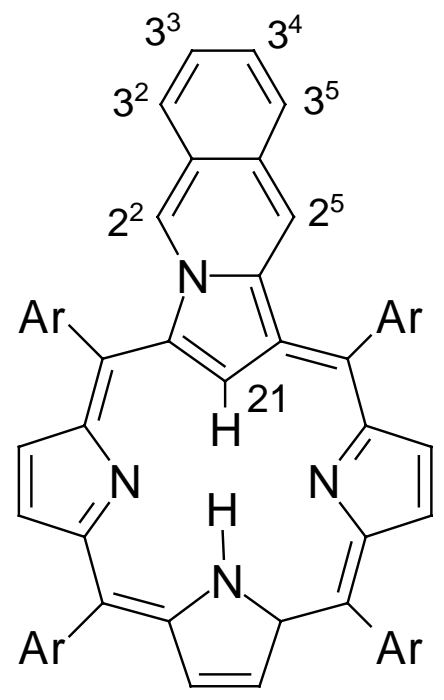




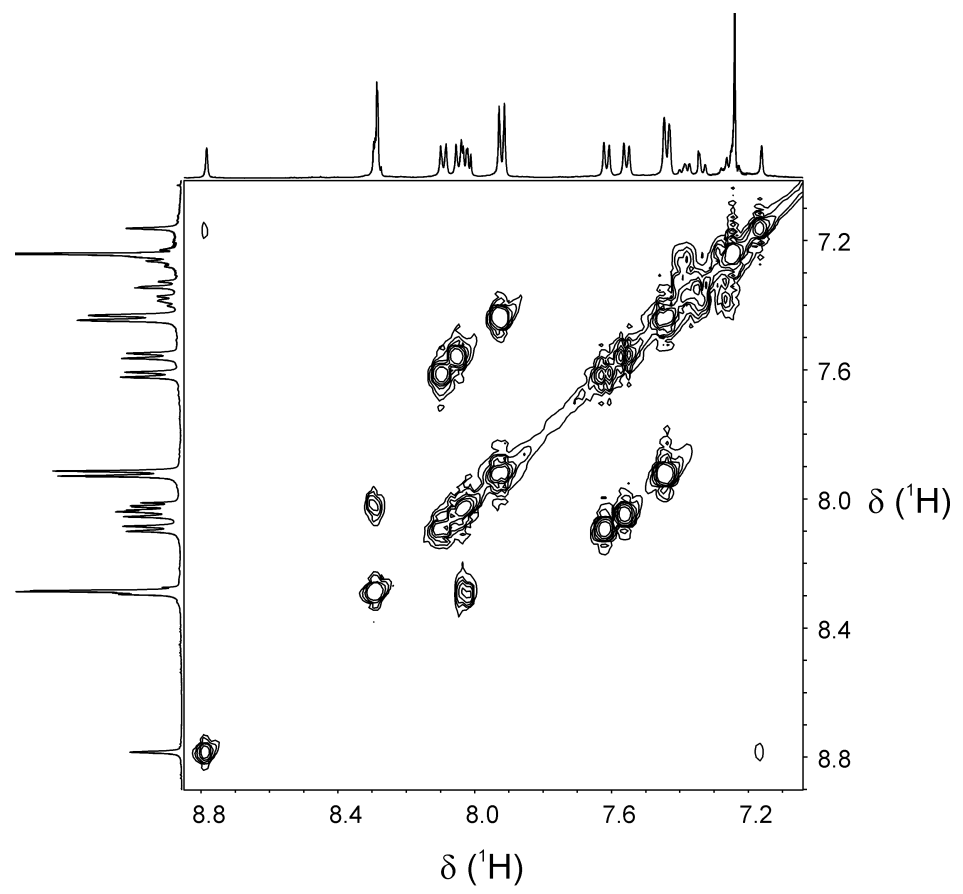

Fig. S6. A fragment of the ${ }^{1} \mathrm{H}-{ }^{1} \mathrm{H}$ COSY spectrum of $5\left(\mathrm{CDCl}_{3}, 298 \mathrm{~K}\right)$

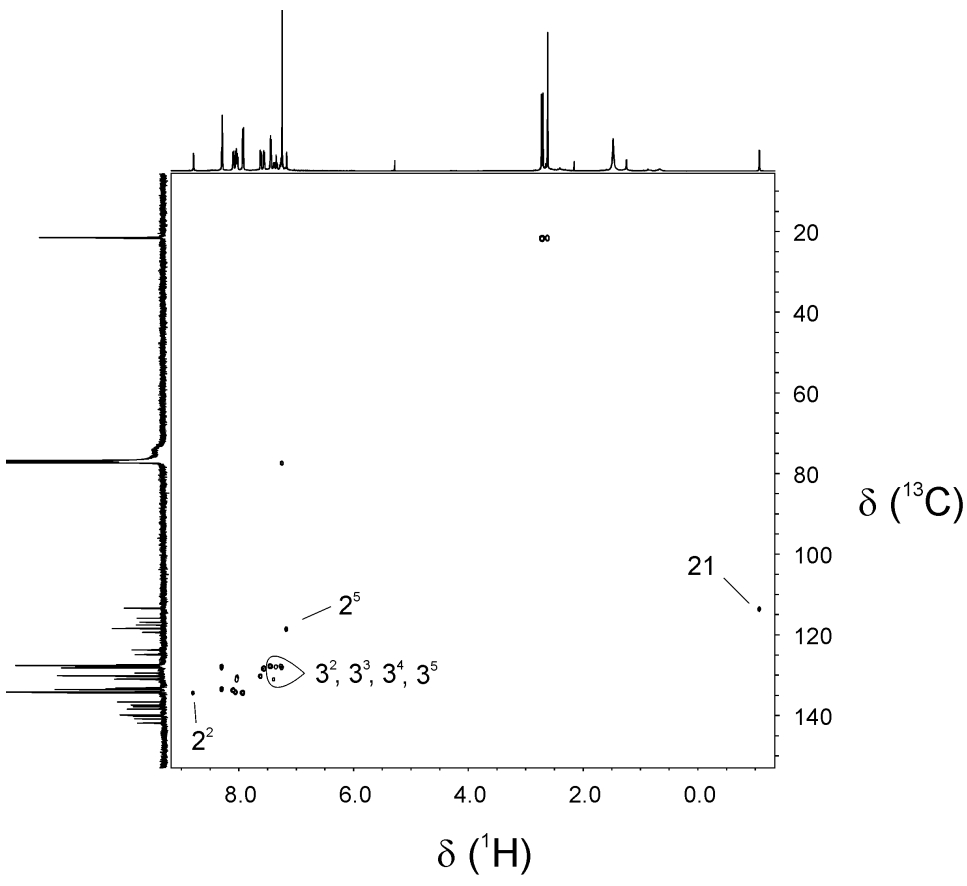

Fig. S7. ${ }^{1} \mathrm{H}-{ }^{13} \mathrm{C}$ HMQC spectrum of $\mathbf{5}, \mathrm{CDCl}_{3}, 298 \mathrm{~K}$. 

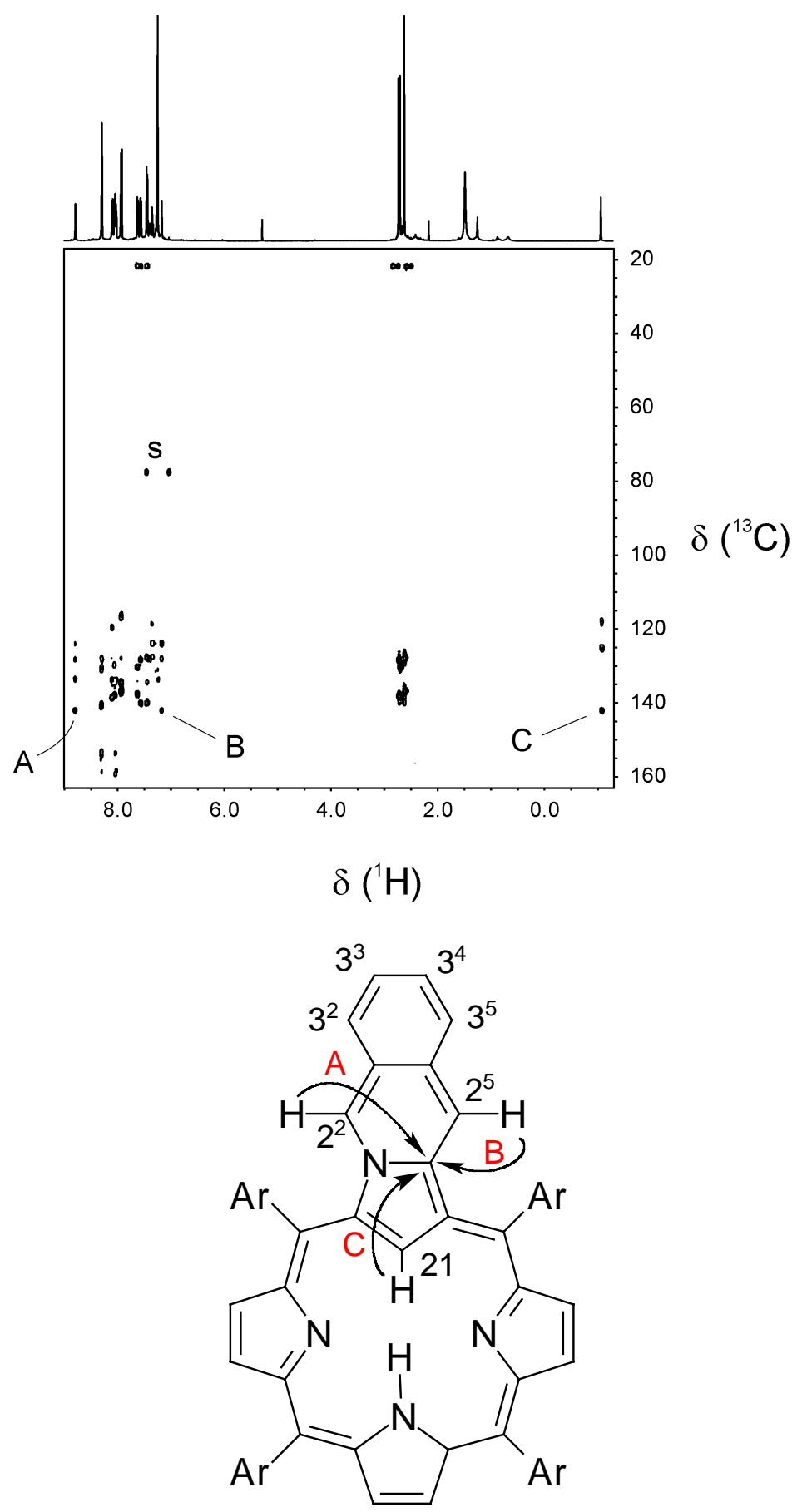

Fig. S8. ${ }^{1} \mathrm{H}_{-}{ }^{13} \mathrm{C}$ HMBC spectrum of $\mathbf{5}, \mathrm{CDCl}_{3}, 298 \mathrm{~K}$. The letters on the map denote correlations between protons and carbon 3 so as they are marked on the scheme. 

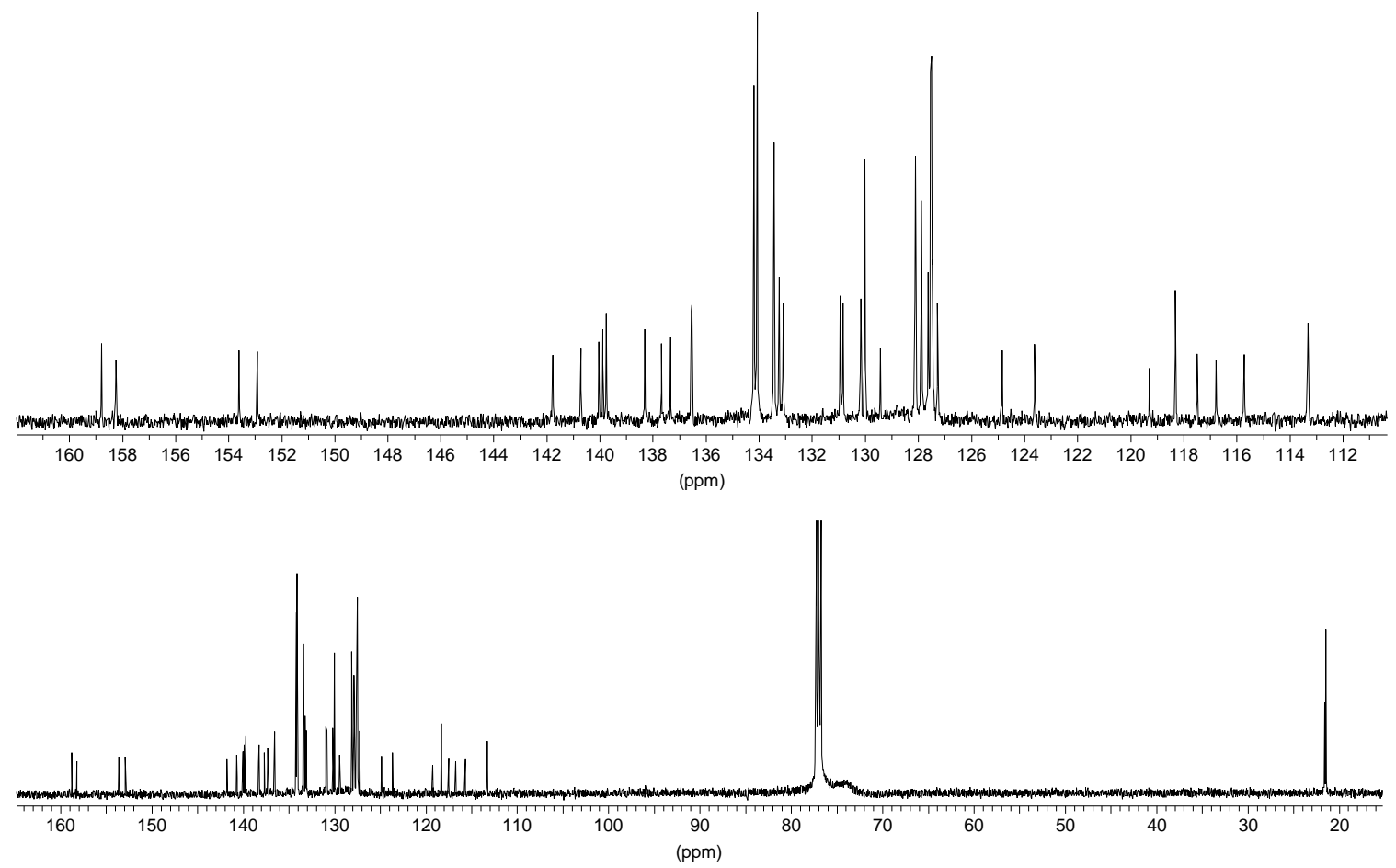

Fig. S9. ${ }^{13} \mathrm{C}$ NMR spectrum of $\mathbf{5}, \mathrm{CDCl}_{3}, 298 \mathrm{~K}$. 

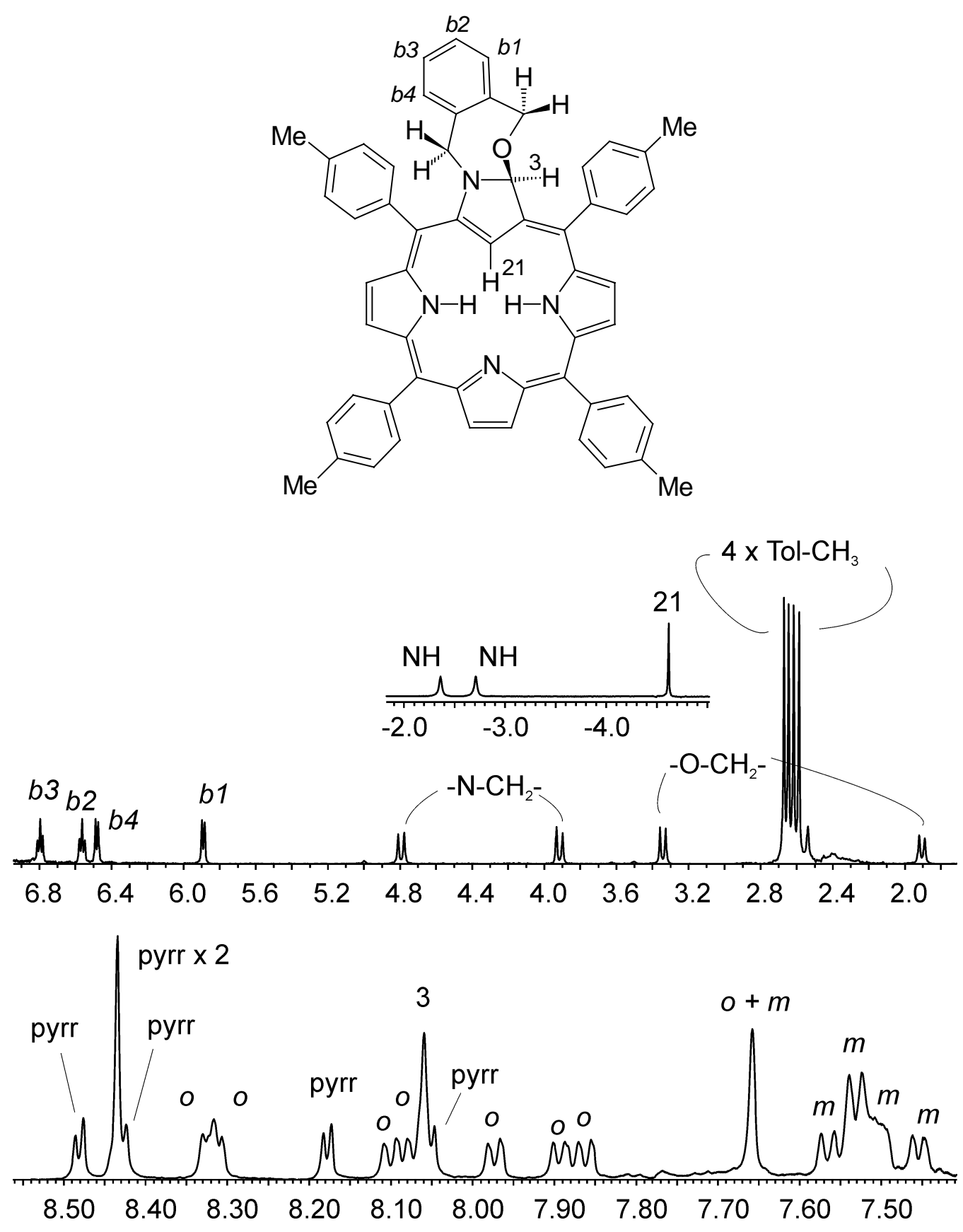

Fig. S10. ${ }^{1} \mathrm{H}$ NMR spectrum of $6, \mathrm{CDCl}_{3}, 213 \mathrm{~K}$. Assignments: pyrr, $\beta$-pyrrole, $o, m$ - ortho and meta protons of meso-tolyl substituents, respectively, $b 1-b 4$, phenylene protons of the xylene fragment.. 


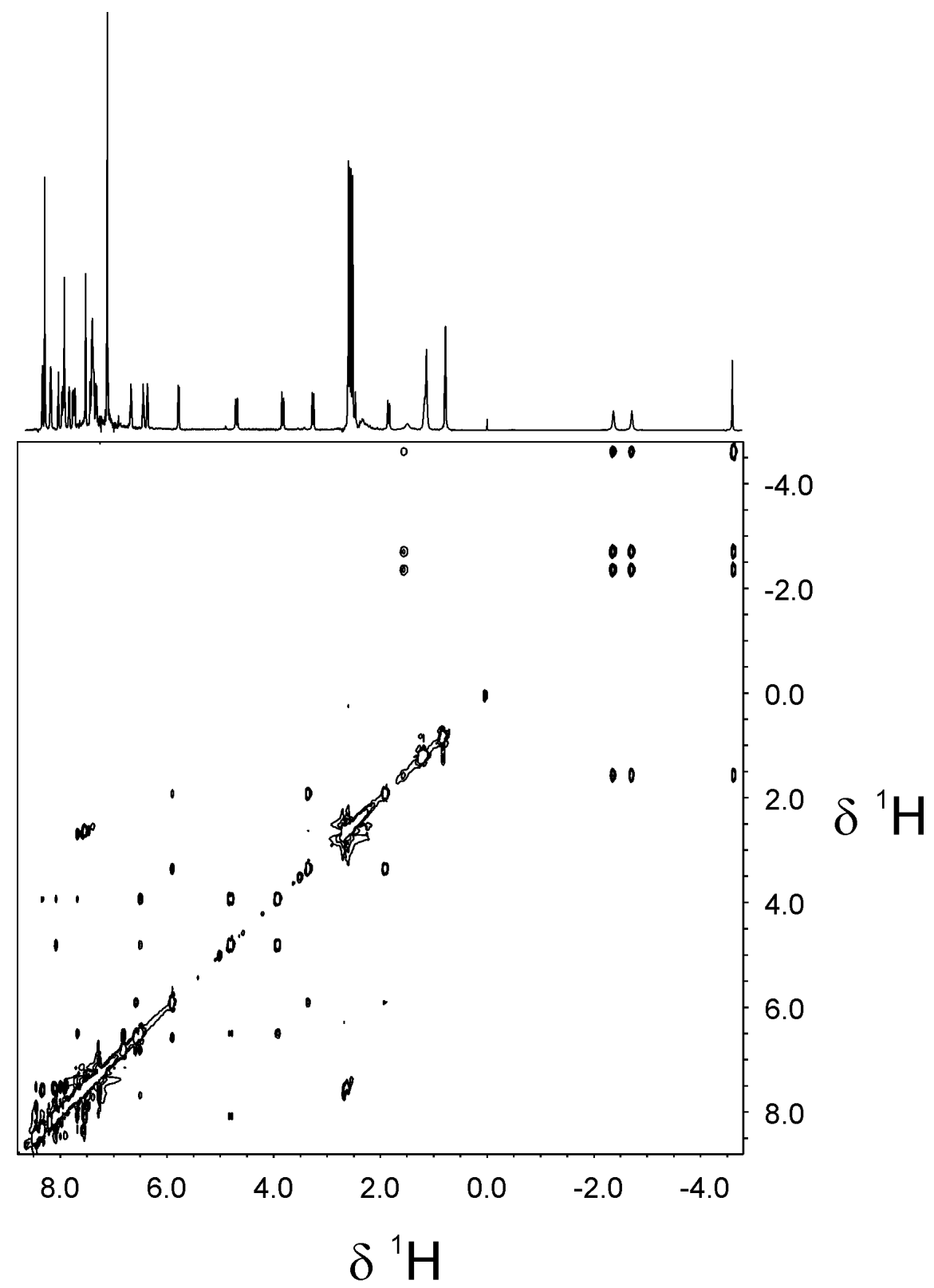

Fig. S11. NOESY spectrum of $\mathbf{6}, 213 \mathrm{~K}, \mathrm{CDCl}_{3}$. 

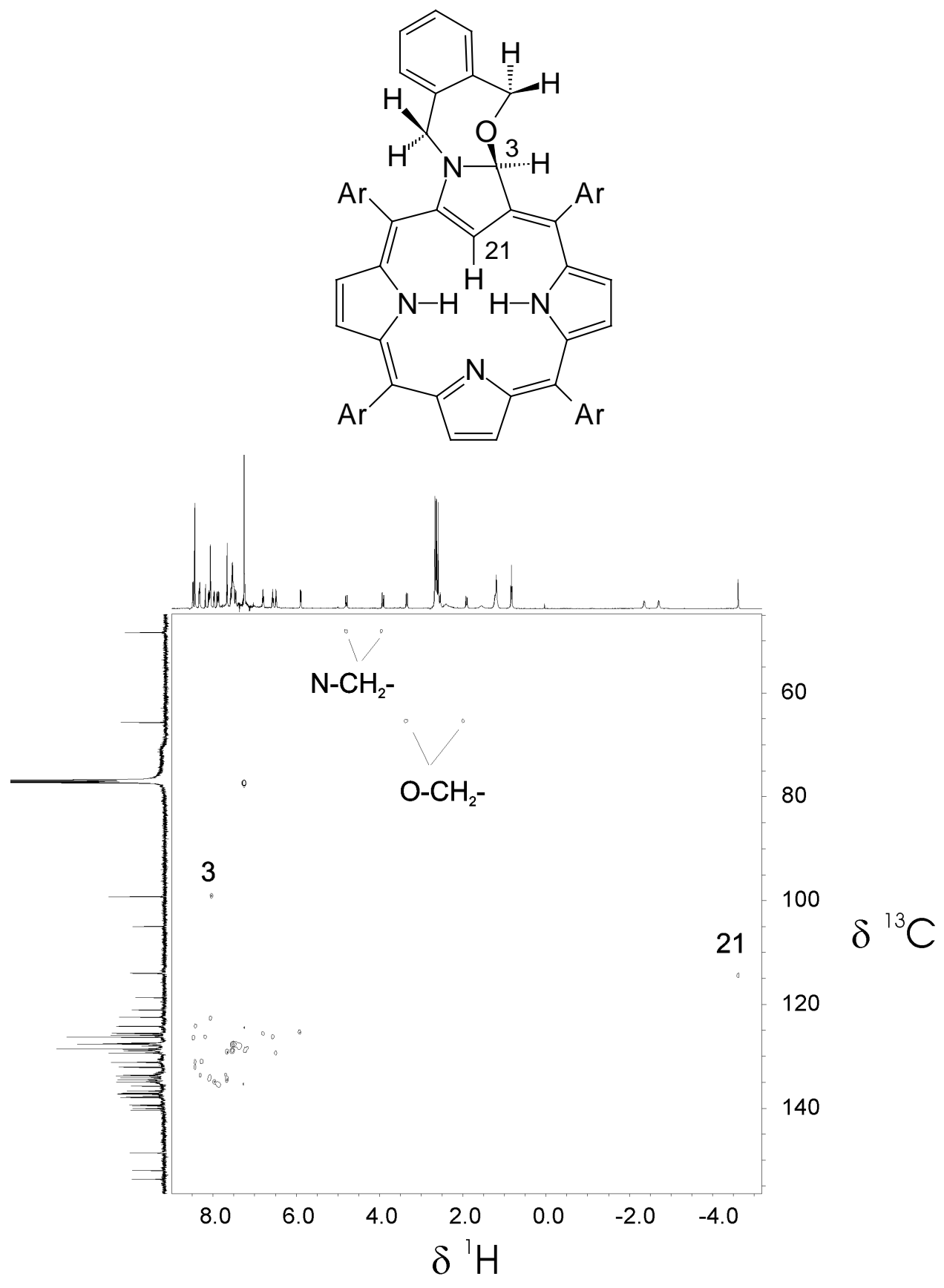

Fig. S12. ${ }^{1} \mathrm{H}-{ }^{13} \mathrm{C}$ HMQC spectrum of $\mathbf{6}, \mathrm{CDCl}_{3}, 213 \mathrm{~K}$. 


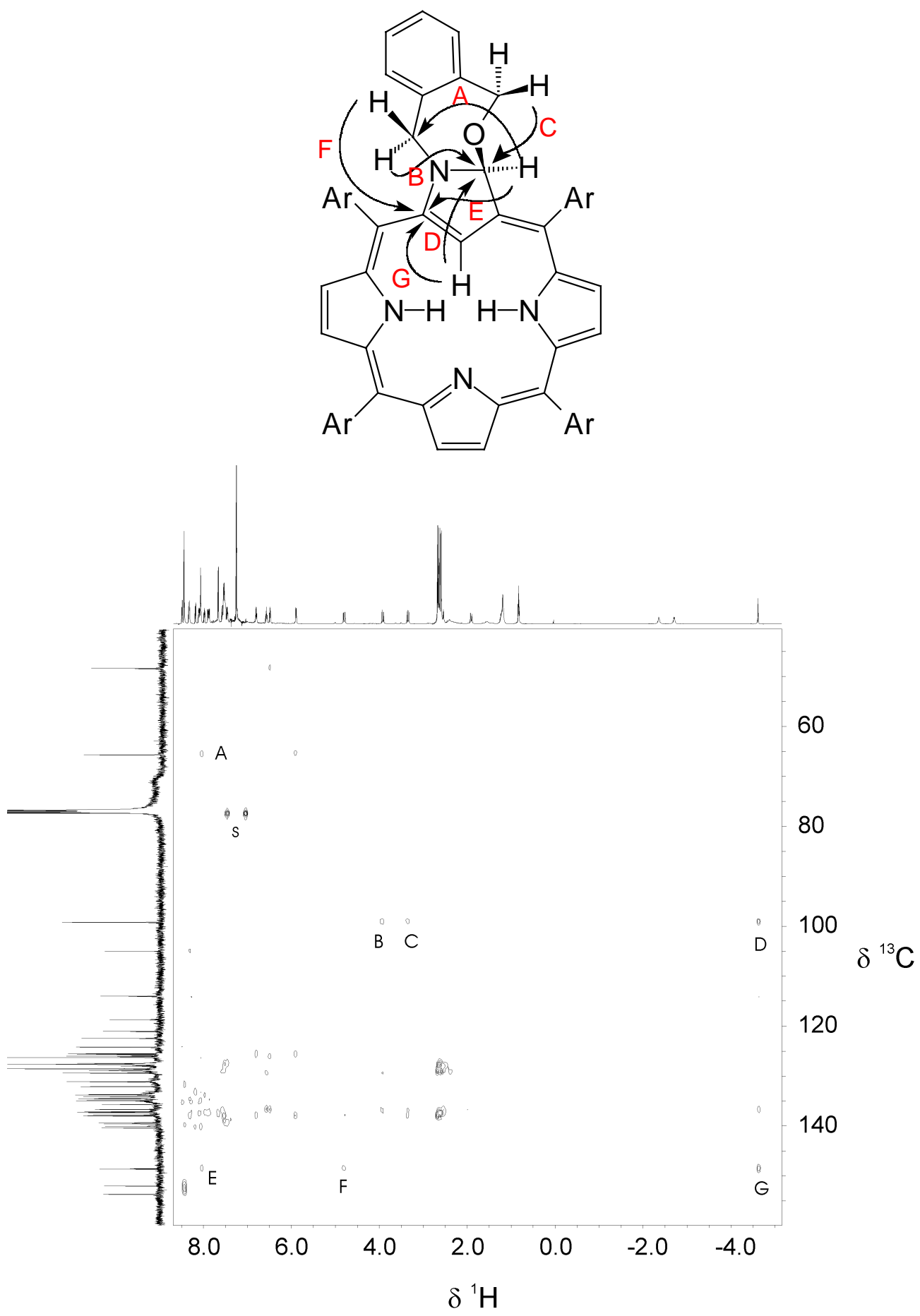

Fig. S13. ${ }^{1} \mathrm{H}_{-}{ }^{13} \mathrm{C}$ HMBC spectrum of $6, \mathrm{CDCl}_{3}, 213 \mathrm{~K}$. The letters on the map denote correlations between protons and carbons marked on the scheme by arrows. 

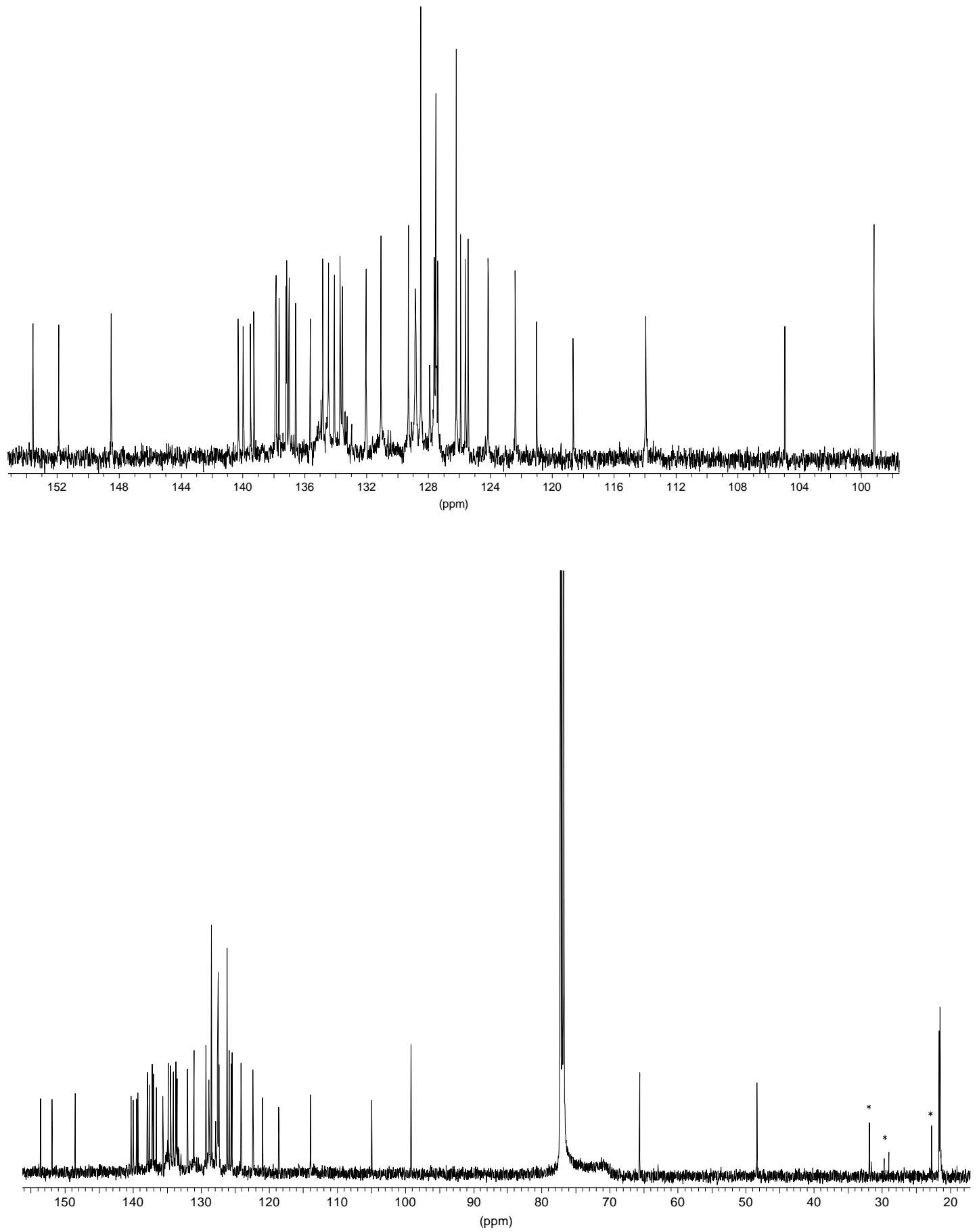

Fig. S14. ${ }^{13} \mathrm{C}$ NMR spectrum of $\mathbf{6}, \mathrm{CDCl}_{3}, 298 \mathrm{~K}$. Signals of impurity (hexane) are marked by asterisk. 


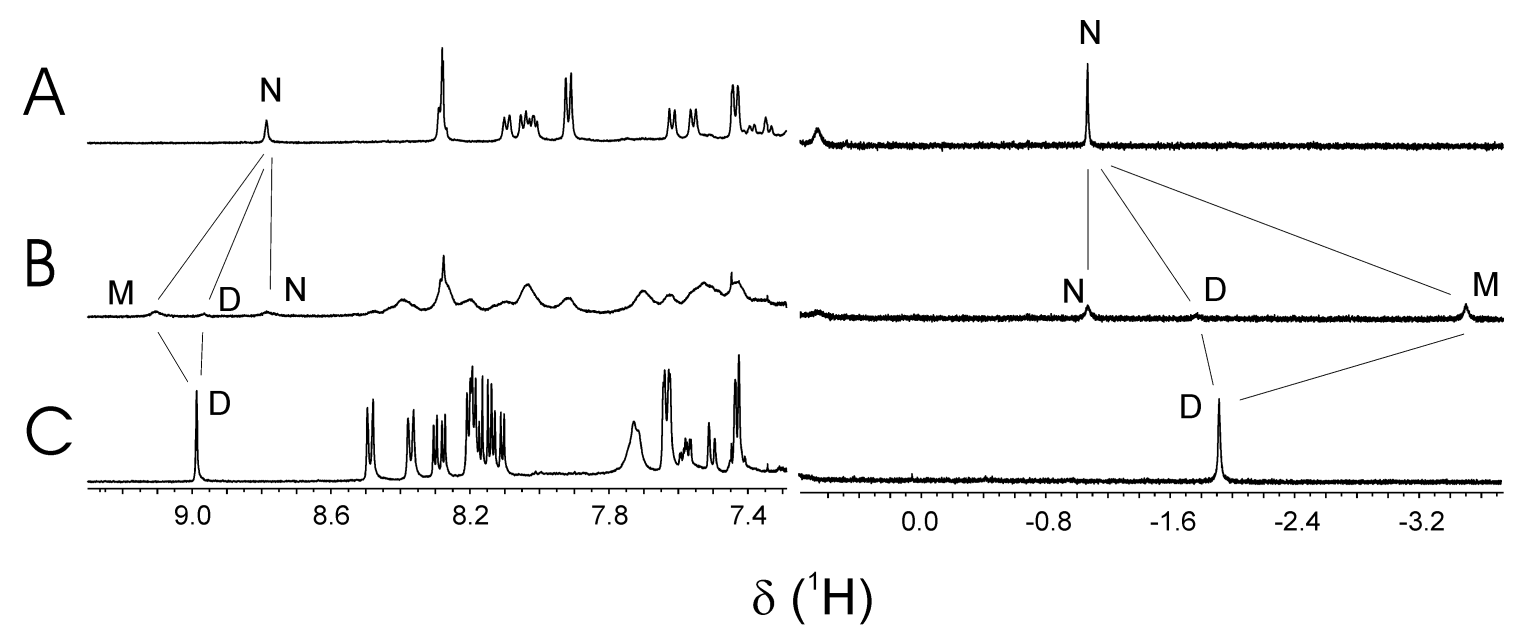

Fig. S15. Low- and high-field parts of the ${ }^{1} \mathrm{H}$ NMR spectra taken upon titration of $\mathbf{5}$ with trifluoroacetic acid $\left(\mathrm{CDCl}_{3}, 298 \mathrm{~K}\right)$ : $\mathrm{A}-0$ equiv.; $\mathrm{B}-1$ equiv.; $\mathrm{C}-3$ equiv. Assignments: $\mathrm{N}$ - signals of neutral macrocycle; $\mathrm{M}$ - signals of monocation; $\mathrm{D}$ - signals of dication.

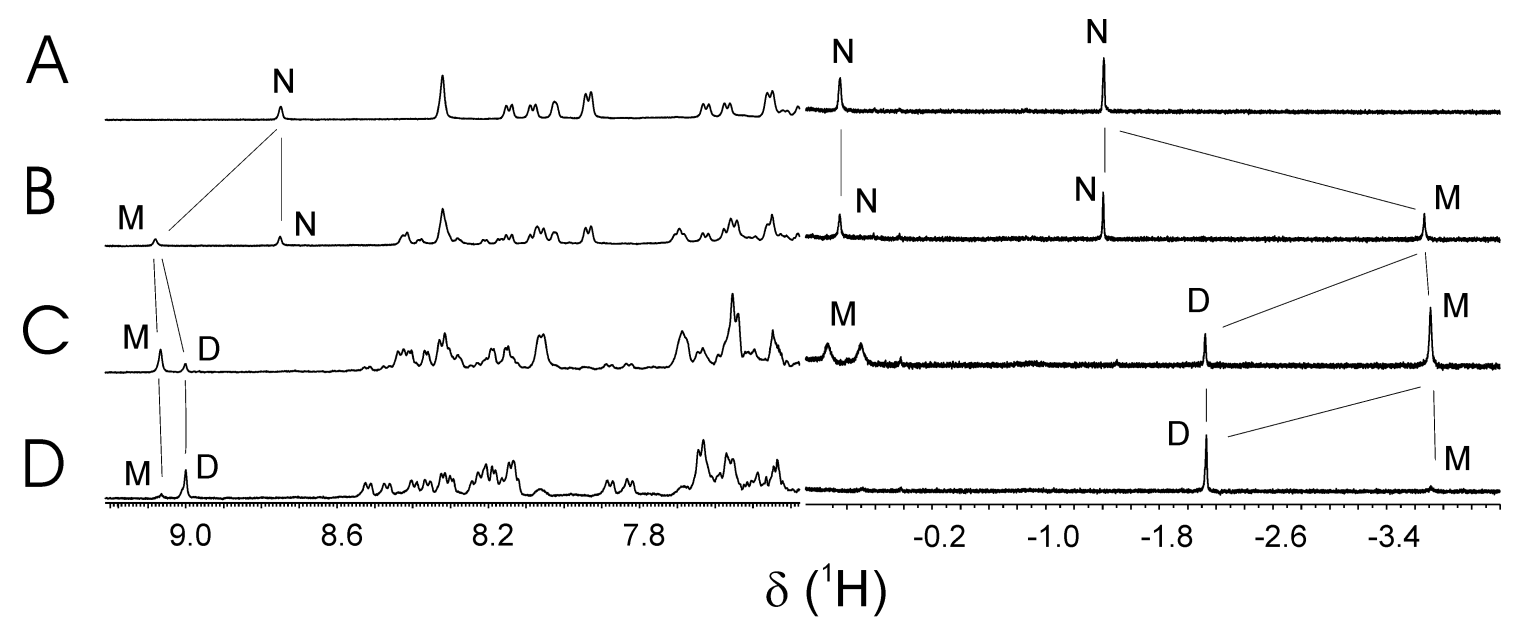

Fig. S16. Low- and high-field parts of the ${ }^{1} \mathrm{H}$ NMR spectra taken upon titration of $\mathbf{5}$ with trifluoroacetic acid $\left(\mathrm{CDCl}_{3}, 213 \mathrm{~K}\right)$ : $\mathrm{A}-0$ equiv.; $\mathrm{B}-0.6$ equiv.; $\mathrm{C}-1$ equiv.; $\mathrm{D}-2$ equiv. Assignments: $\mathrm{N}$ - signals of neutral macrocycle; $\mathrm{M}$ - signals of monocation; $\mathrm{D}$ - signals of dication. 


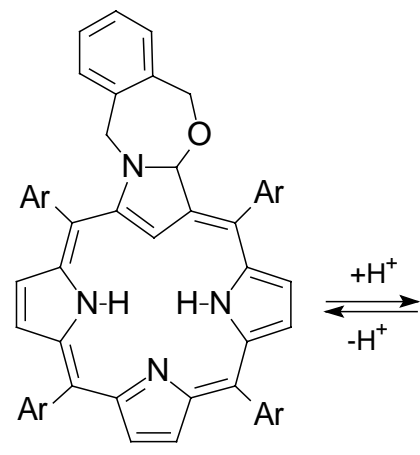

6

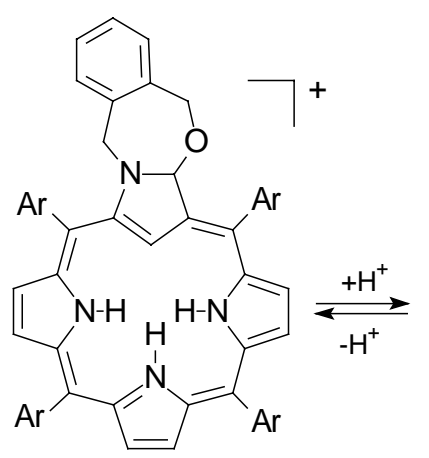

$[6-\mathrm{H}]^{+}$

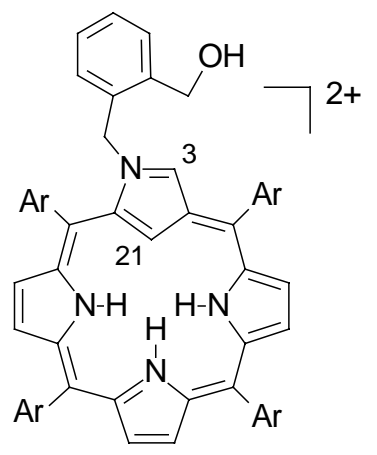

$\left[6-\mathrm{H}_{2}\right]^{2+}$

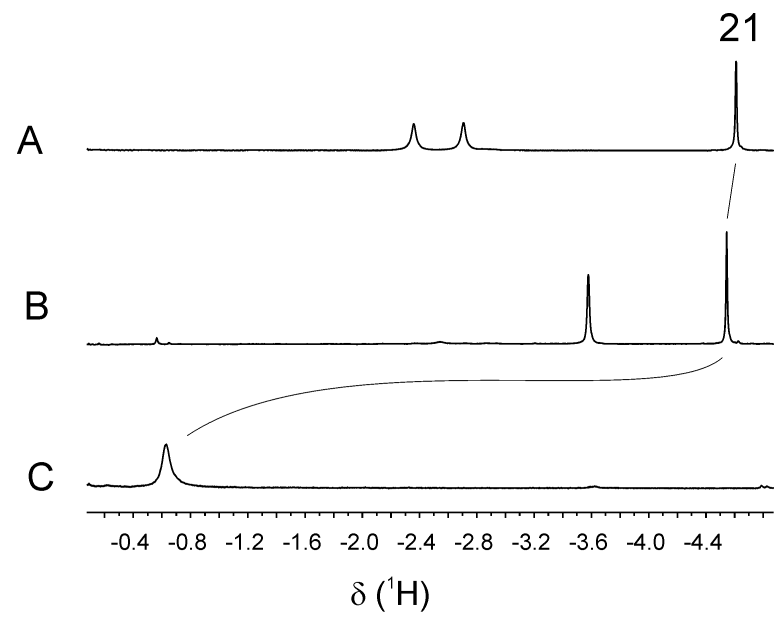

Fig. S17. High-field fragments of the ${ }^{1} \mathrm{H}$ NMR spectra $\left(\mathrm{CDCl}_{3}, 213 \mathrm{~K}\right)$ of $\mathbf{6}$ (A), its monokation $[6-\mathrm{H}]^{+}(\mathrm{B})$, and dication $\left[6-\mathrm{H}_{2}\right]^{2+}(\mathrm{C})$.

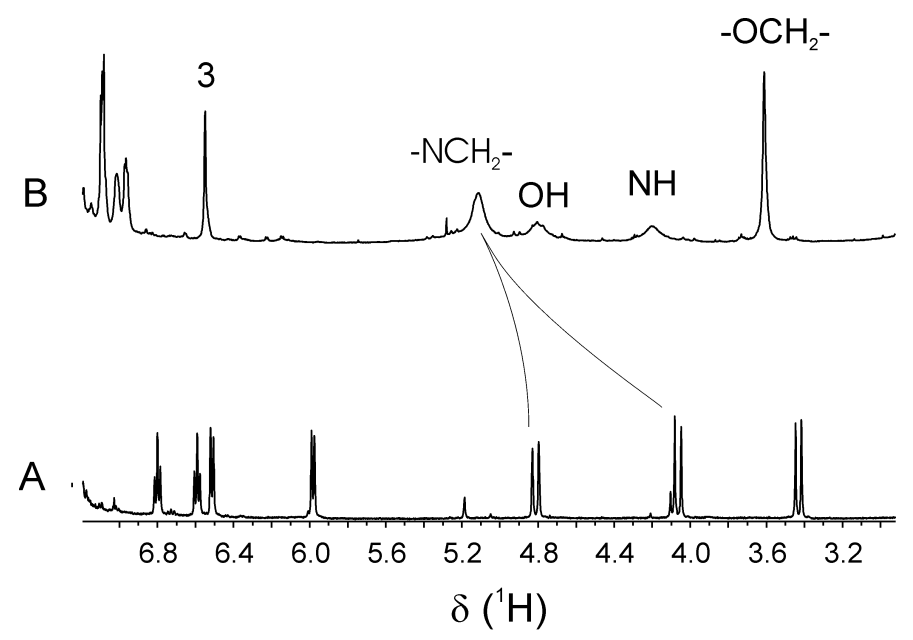

Fig. S18. Fragments of the ${ }^{1} \mathrm{H}$ NMR spectra $\left(\mathrm{CDCl}_{3}, 298 \mathrm{~K}\right)$ of $\mathbf{6}(\mathrm{A})$, and dication $\left[6-\mathrm{H}_{2}\right]^{2+}$ (B). 


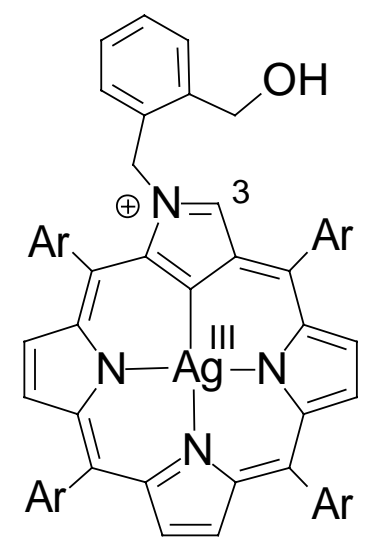

7

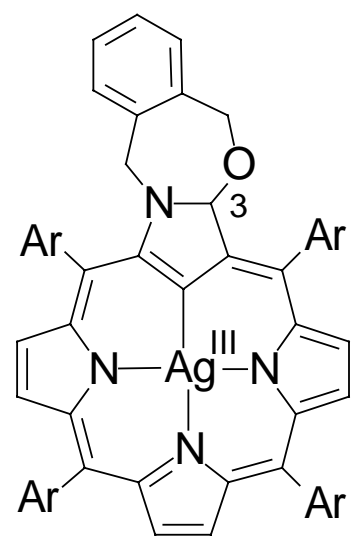

8

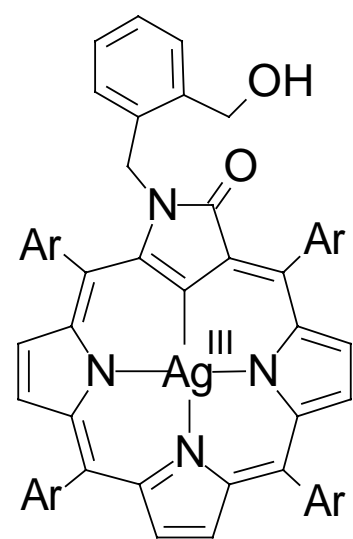

10
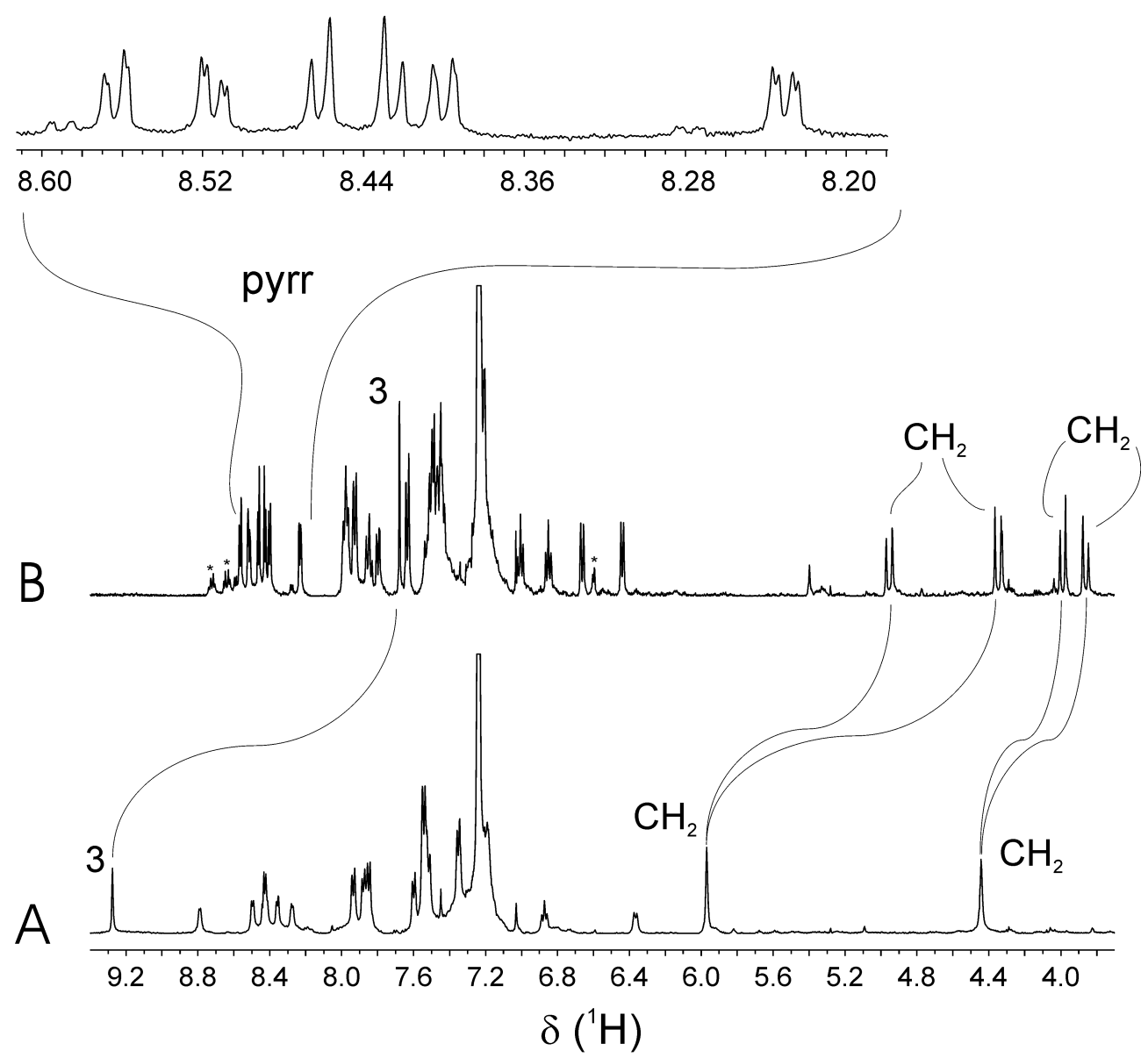

Fig. S19. ${ }^{1} \mathrm{H}$ NMR spectra $\left(\mathrm{CDCl}_{3}, 298 \mathrm{~K}\right)$ of 7 (A) and 8 (B). The inset in B shows extension of a $\beta$-pyrrole region with proton-silver coupling structures seen on some of the signals. The signals marked with asterisks are those of the forming complex $\mathbf{1 0 .}$ 

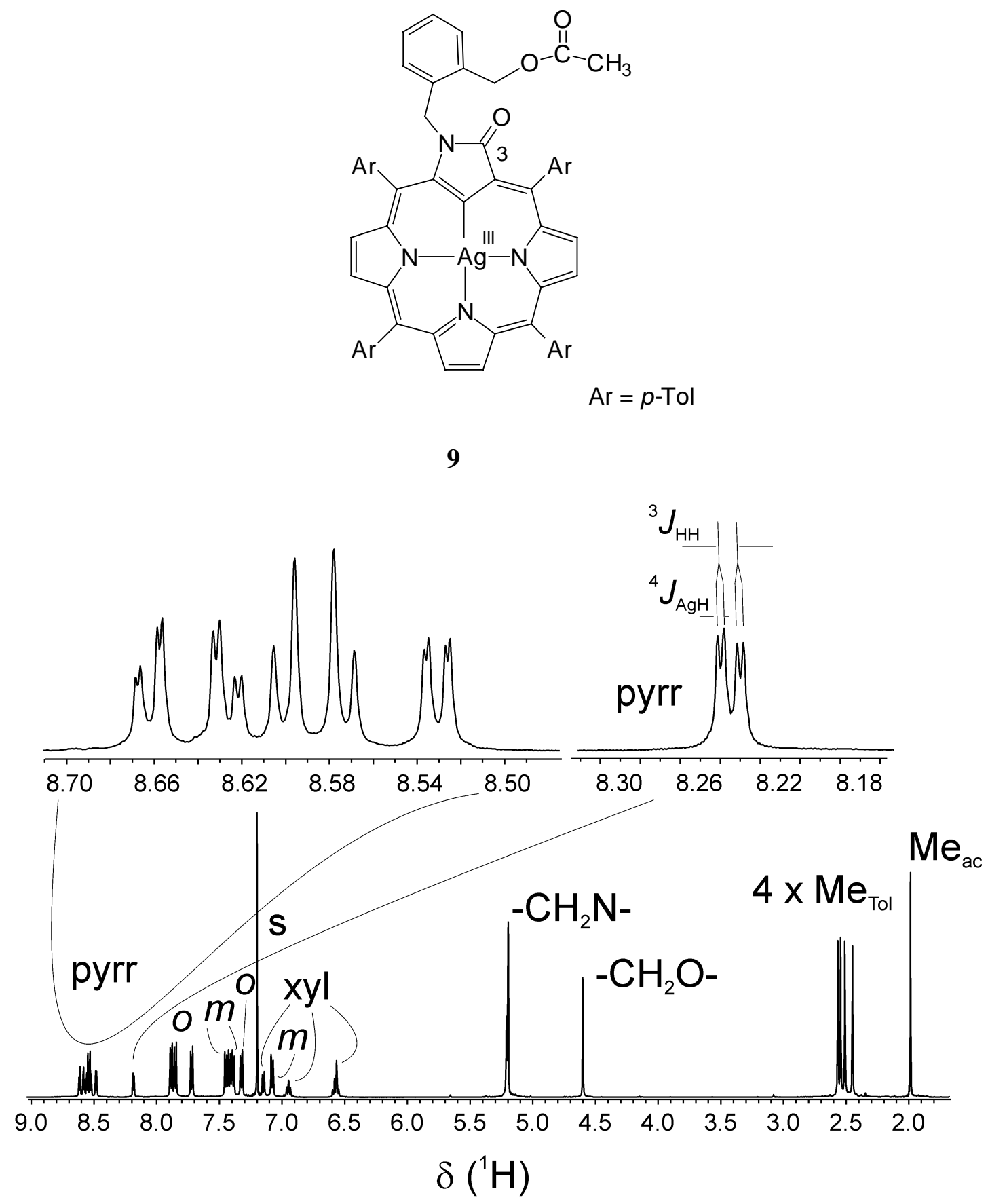

Fig. S20. ${ }^{1} \mathrm{H}$ NMR spectrum $\left(\mathrm{CDCl}_{3}, 298 \mathrm{~K}\right)$ of 9. The inset shows extension of a $\beta$-pyrrole region with proton-silver coupling structures seen on some of the signals. Assignments: pyrr, $\beta$-pyrrole, xyl, phenylene protons of the $o$-xylene fragment, $o, m$ - ortho and meta protons of the meso-tolyl substituent, $\mathrm{Me}_{\mathrm{Tol}}$ - para-methyls of meso-tolyl substituents, $\mathrm{Me}_{\mathrm{ac}}-$ acetate methyl. 

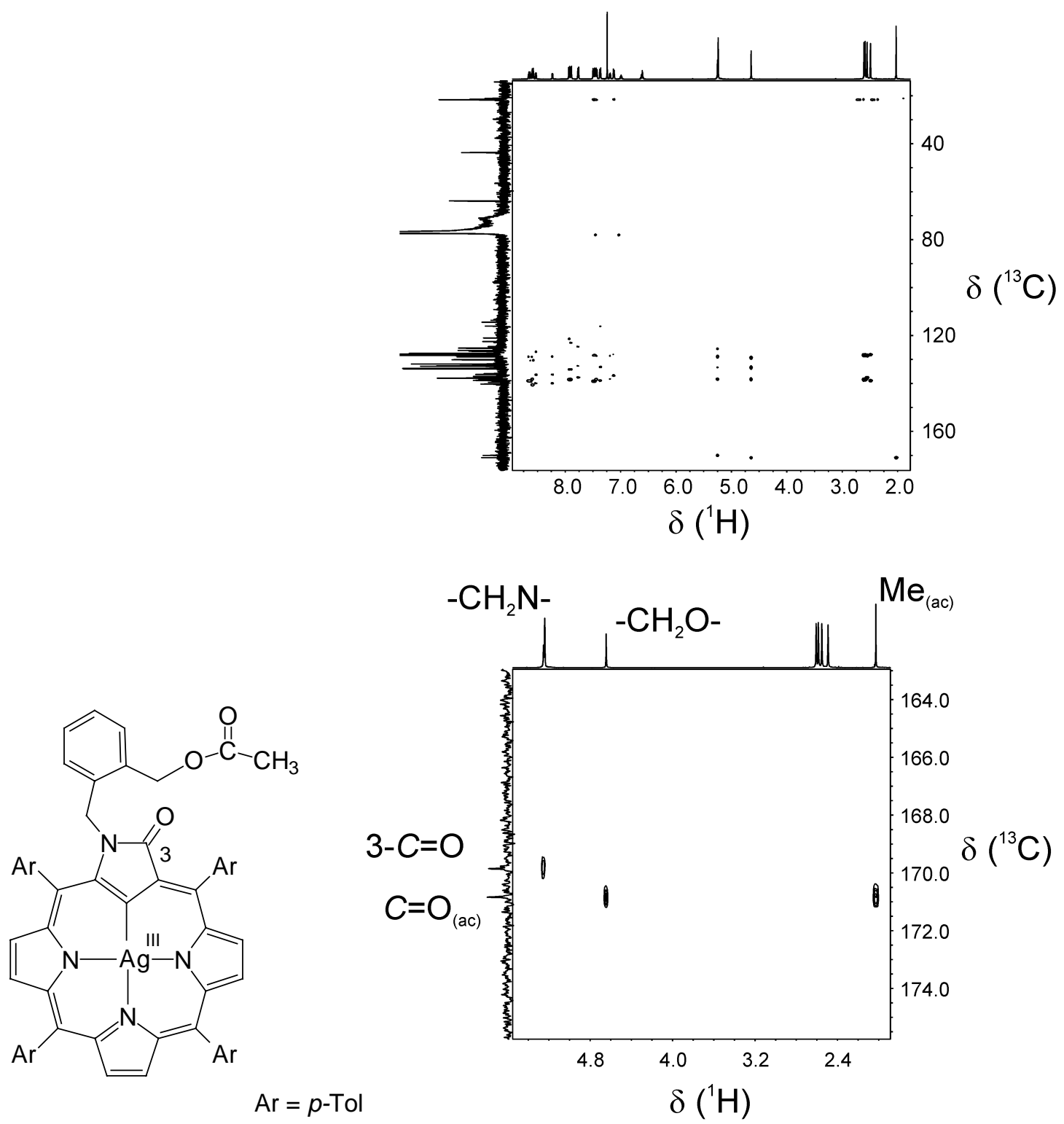

Fig. S21. ${ }^{1} \mathrm{H}_{-}{ }^{13} \mathrm{C}$ HMBC spectrum of 9, $\mathrm{CDCl}_{3}, 298 \mathrm{~K}$ (top) and its fragment (bottom) showing proton correlations with the carbonyl carbons. 


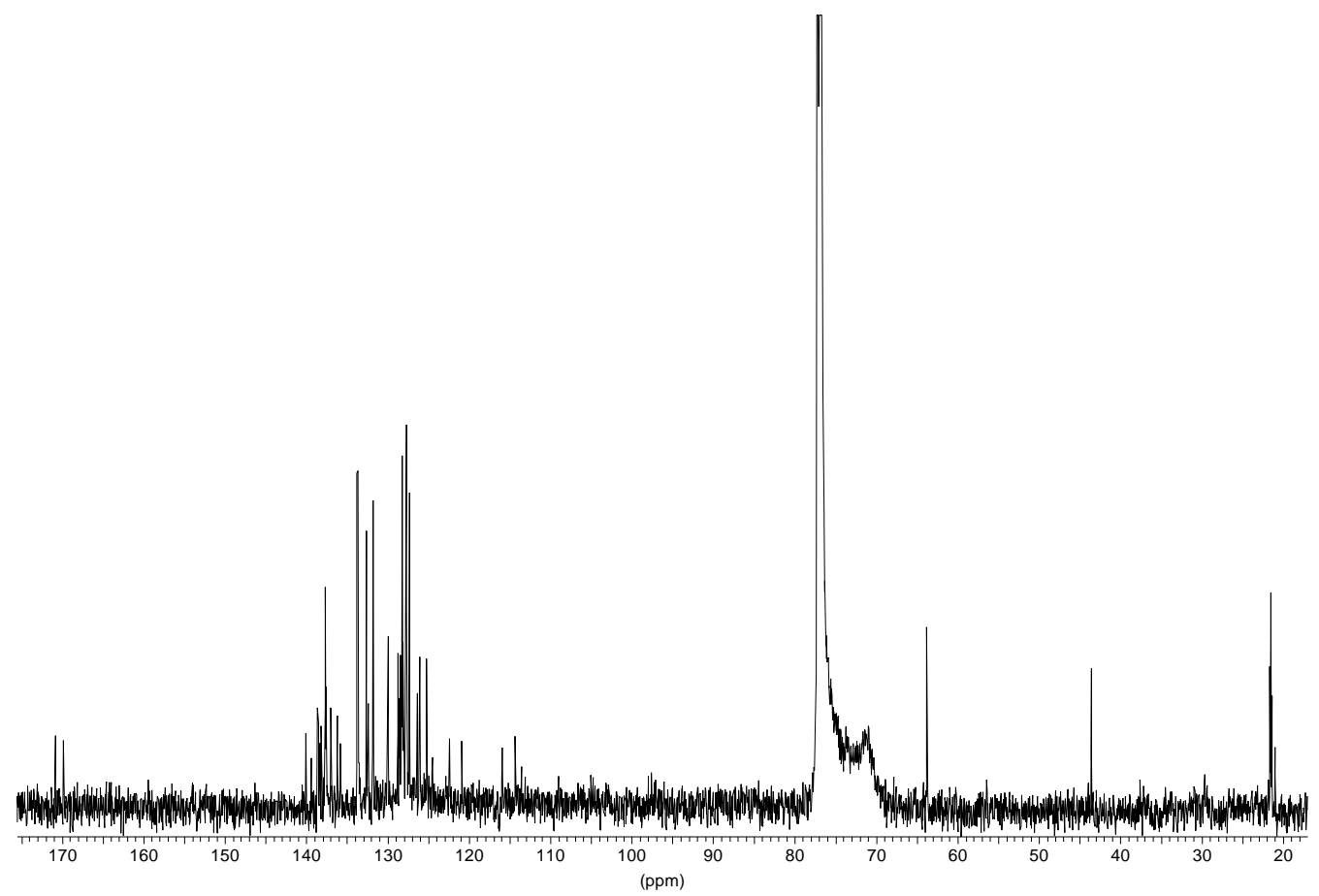

Fig. S22. ${ }^{13} \mathrm{C}$ NMR spectrum of $\mathbf{9}, \mathrm{CDCl}_{3}, 298 \mathrm{~K}$.

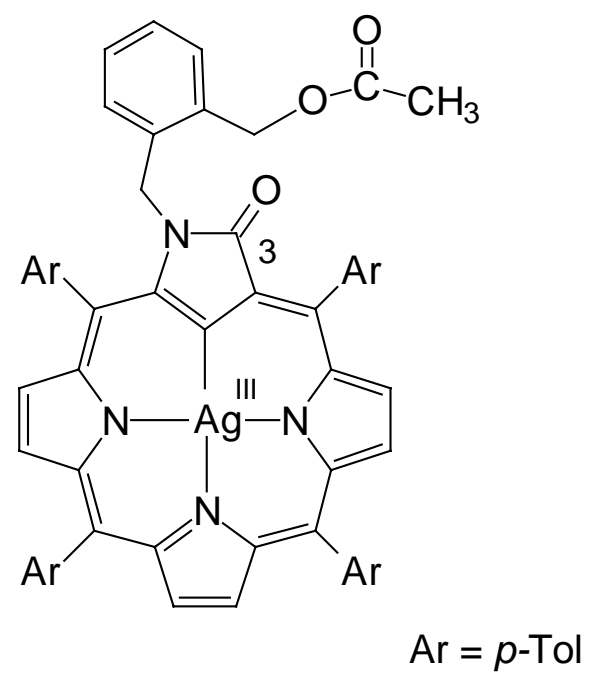




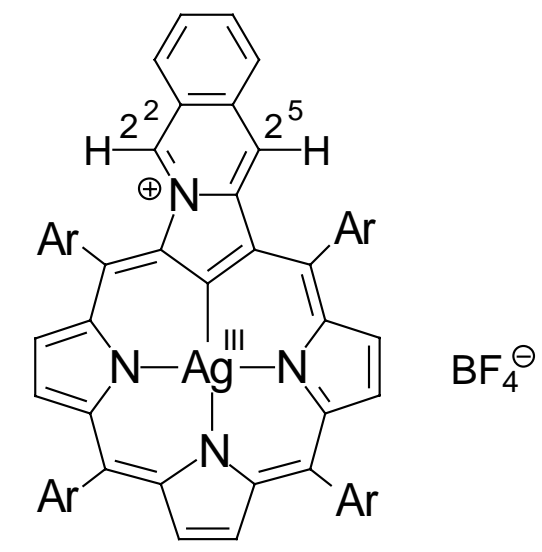

$\operatorname{Ar}=p-$ Tol

11
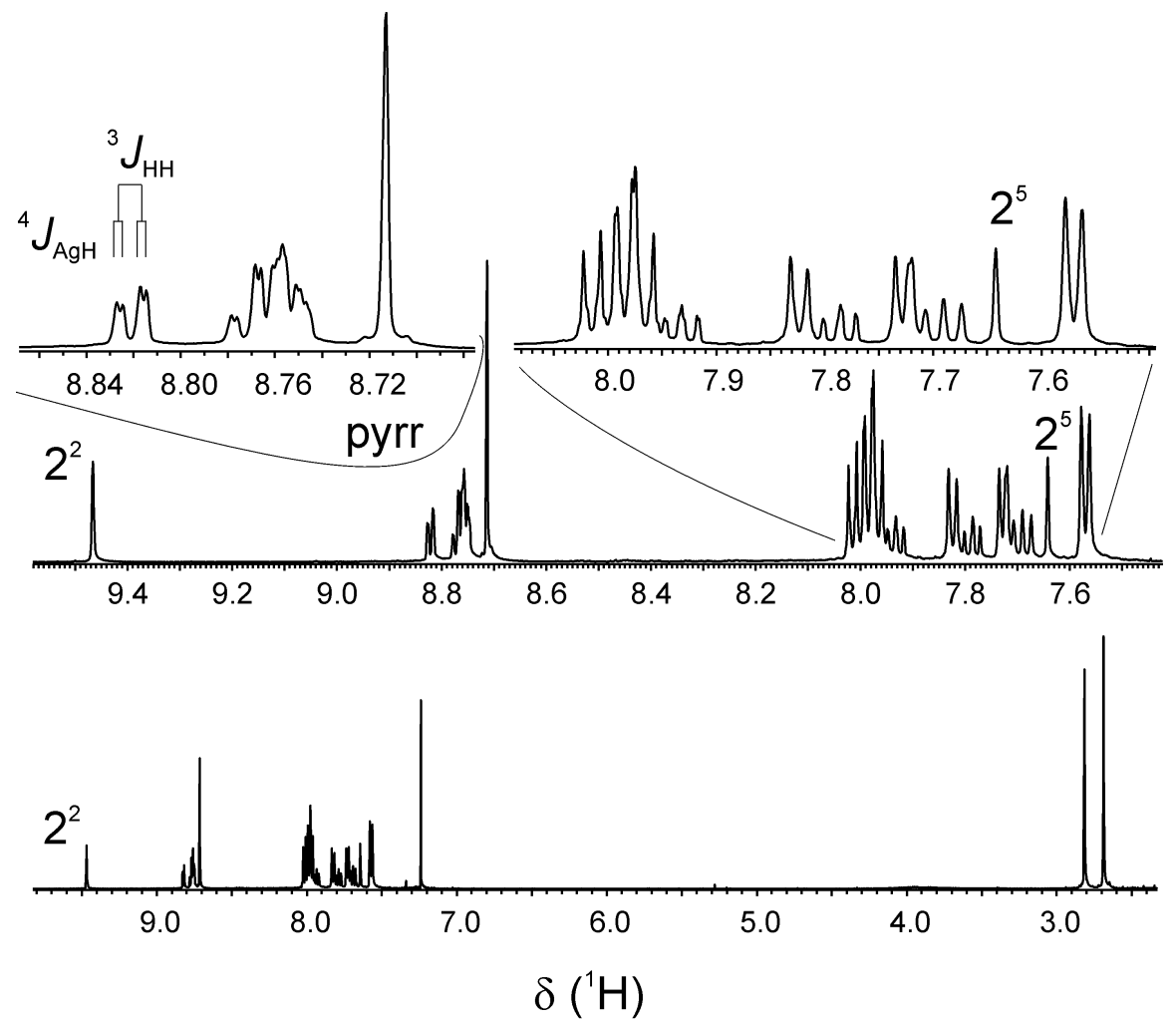

Fig. S23. ${ }^{1} \mathrm{H}$ NMR spectrum $\left(\mathrm{CDCl}_{3}, 298 \mathrm{~K}\right)$ of 11. The top left inset shows extension of a $\beta$ pyrrole region with proton-silver coupling structures seen on some of the signals. 


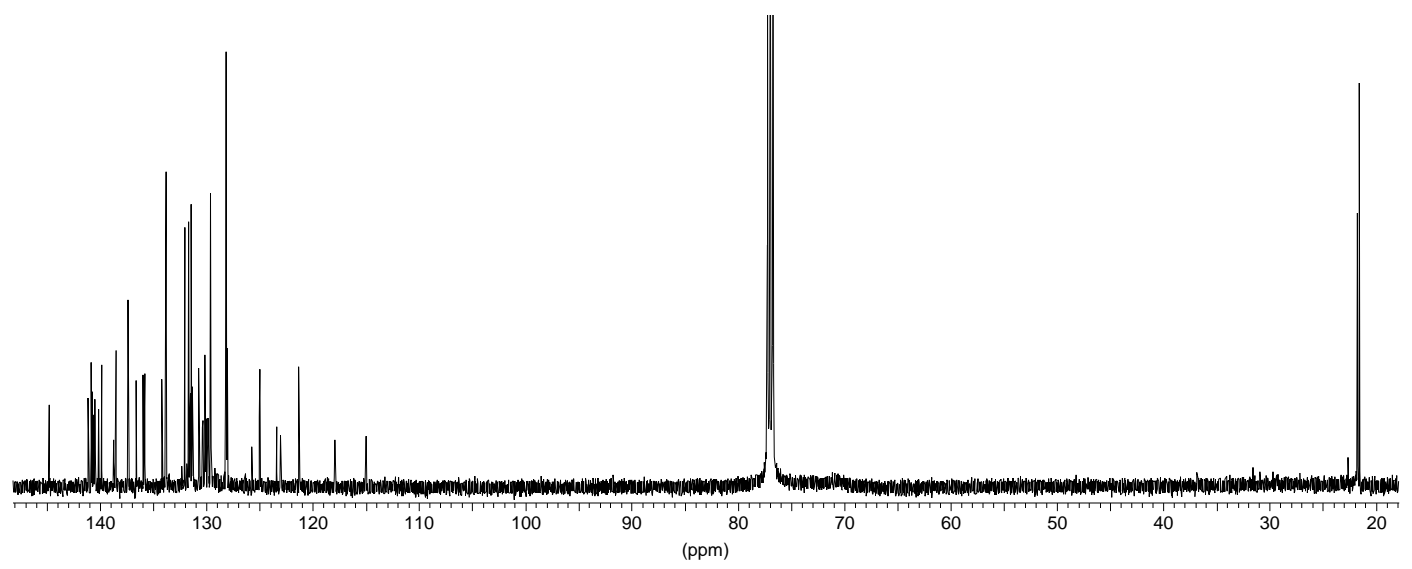

Fig. S24. ${ }^{13} \mathrm{C}$ NMR spectrum of 11, $\mathrm{CDCl}_{3}, 298 \mathrm{~K}$.

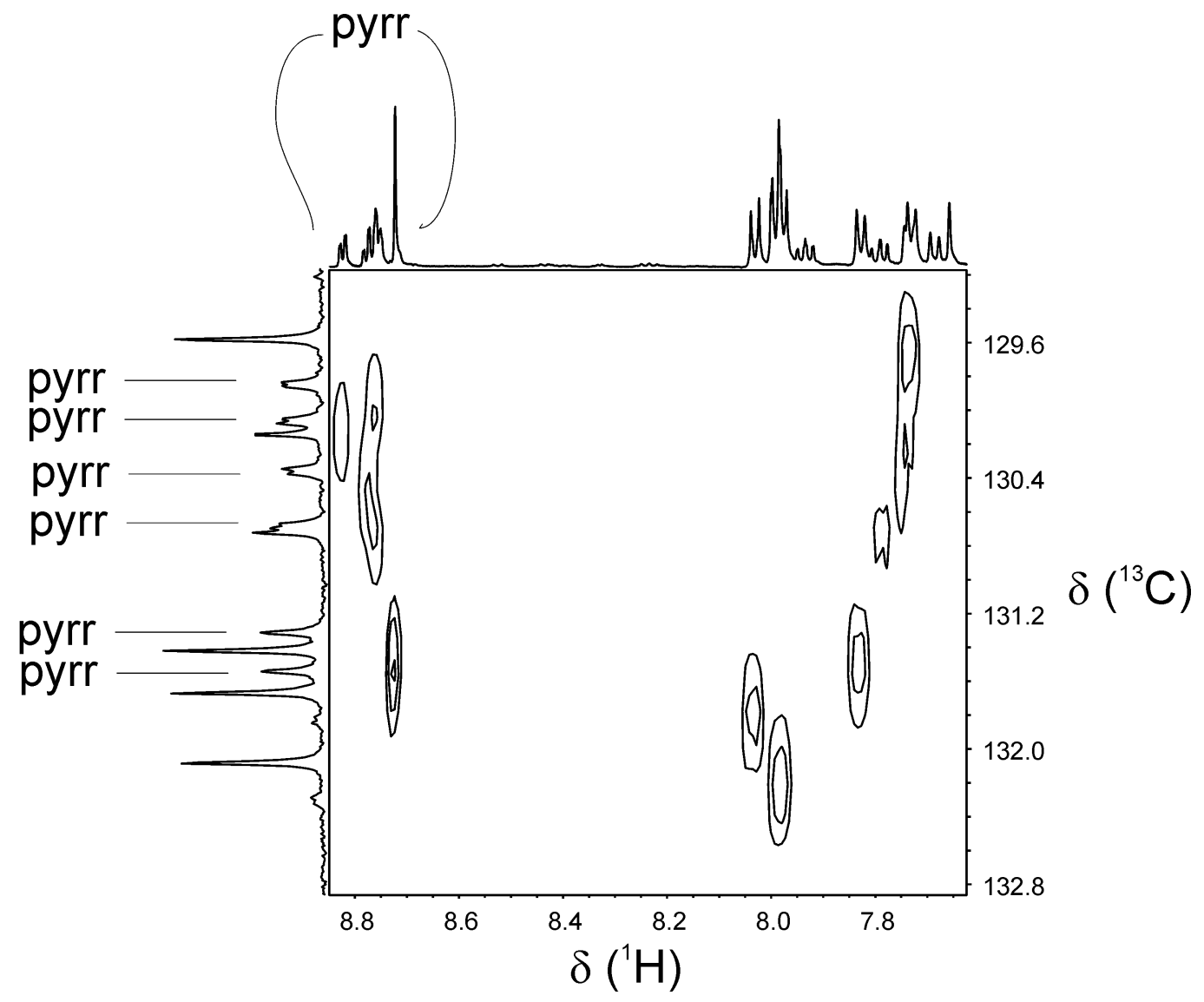

Fig. S25. A fragment of the ${ }^{1} \mathrm{H}^{13} \mathrm{C}$ HMQC spectrum of $11\left(\mathrm{CDCl}_{3}, 298 \mathrm{~K}\right)$ shows correlations between $\beta$-pyrrole protons and carbons. 

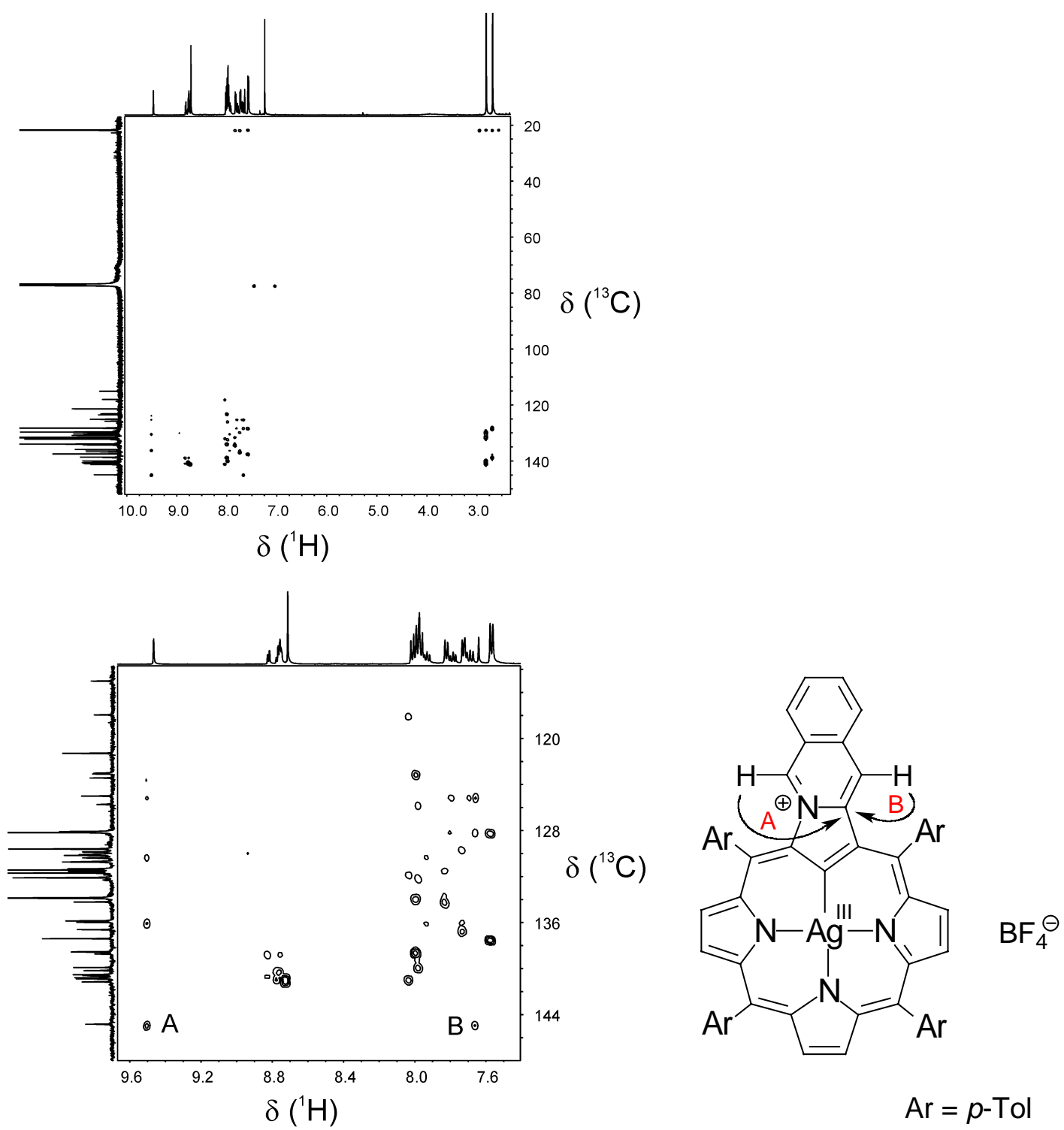

Fig. S26. ${ }^{1} \mathrm{H}_{-}{ }^{13} \mathrm{C} \mathrm{HMBC}$ spectrum of $\mathbf{1 1}, \mathrm{CDCl}_{3}, 298 \mathrm{~K}$ (top) and its fragment (bottom) with marked correlations of carbon 3 with exocyclic ring protons.

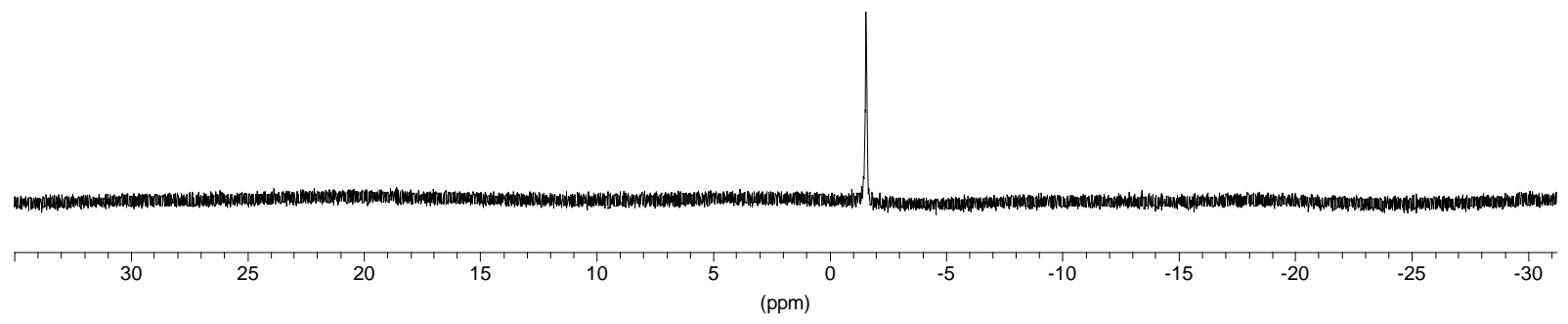

Fig. S27. ${ }^{11} \mathrm{~B}$ NMR spectrum of $\mathbf{1 1}, \mathrm{CDCl}_{3}, 298 \mathrm{~K}$. 


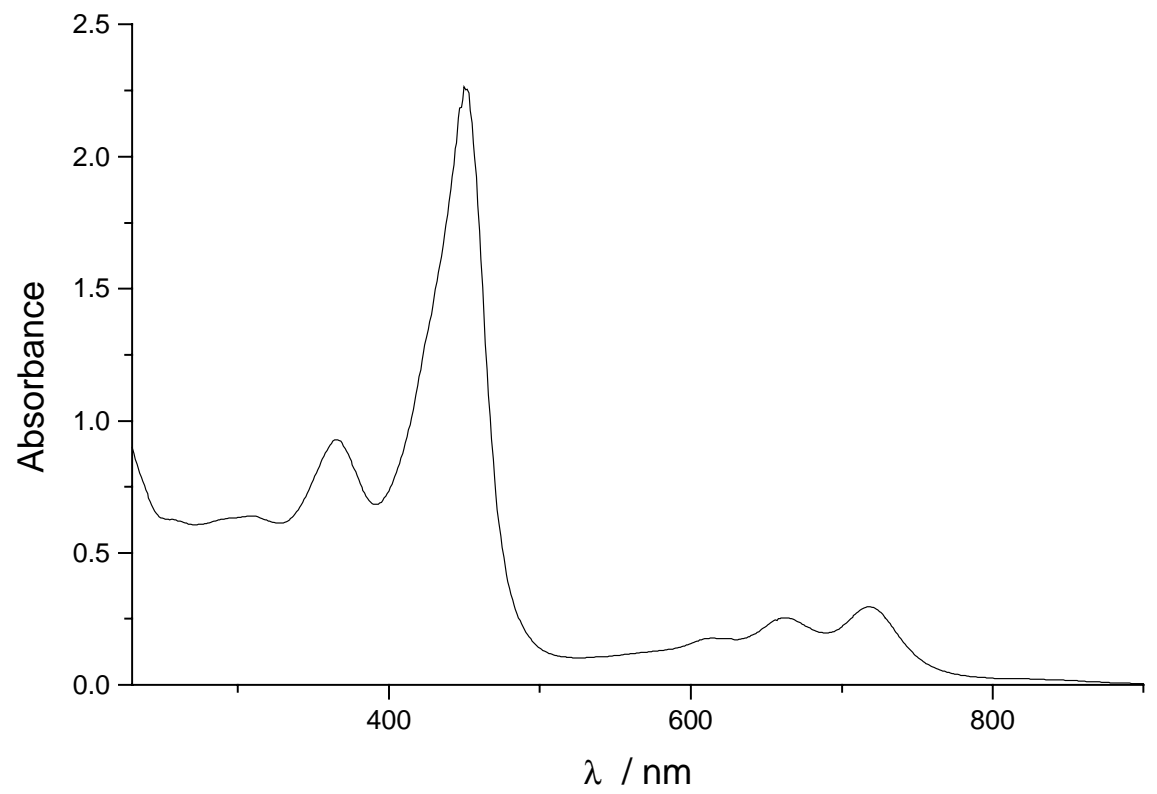

Fig. S28. Optical spectrum of $\mathbf{4}$ in dichloromethane.

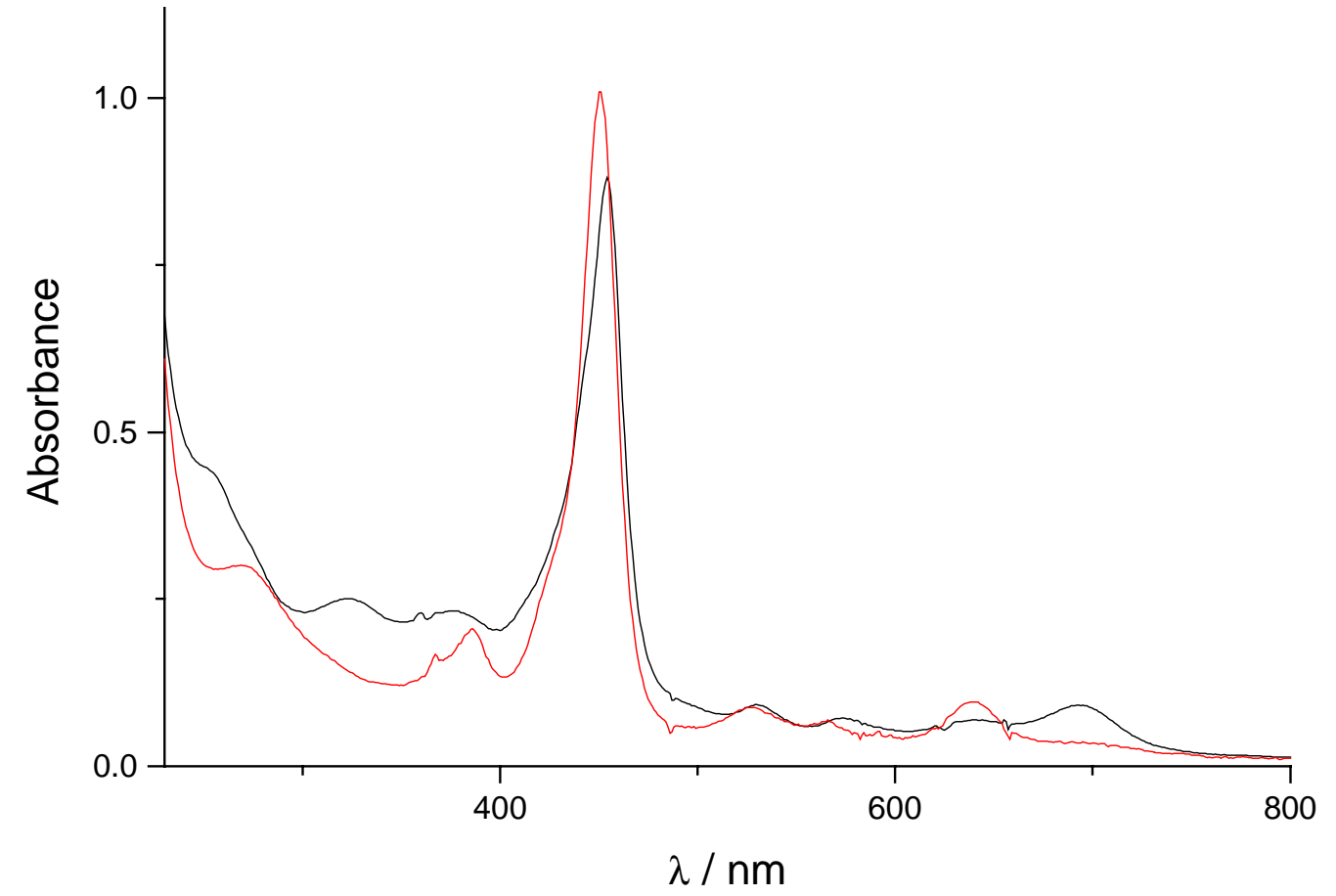

Fig. S29. Optical spectra of $\mathbf{7}$ (black trace) and $\mathbf{8}$ (red trace) in dichloromethane. 


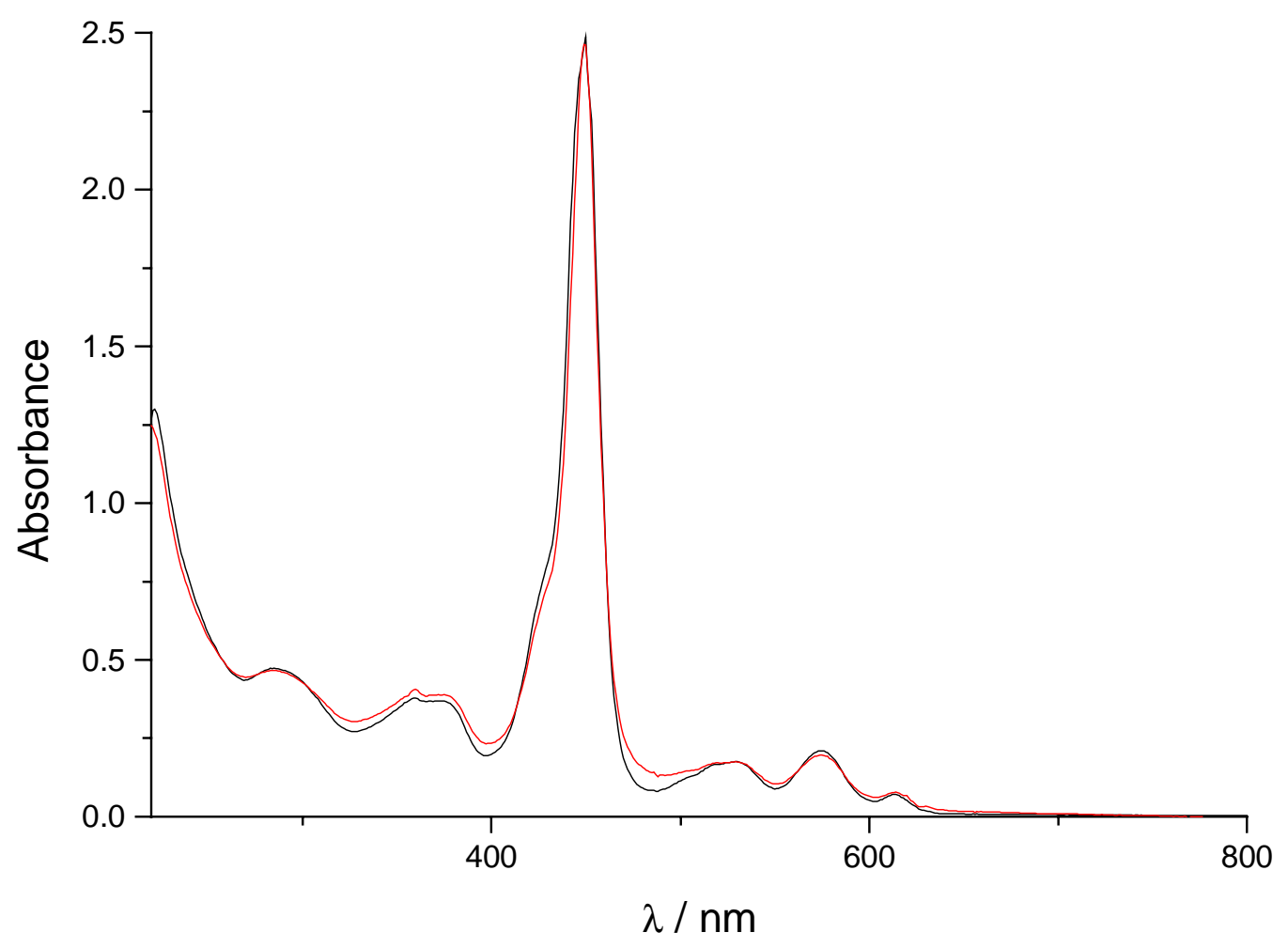

Fig. S30. Optical spectra of $\mathbf{9}$ (black trace) and $\mathbf{1 0}$ (red trace) in dichloromethane.

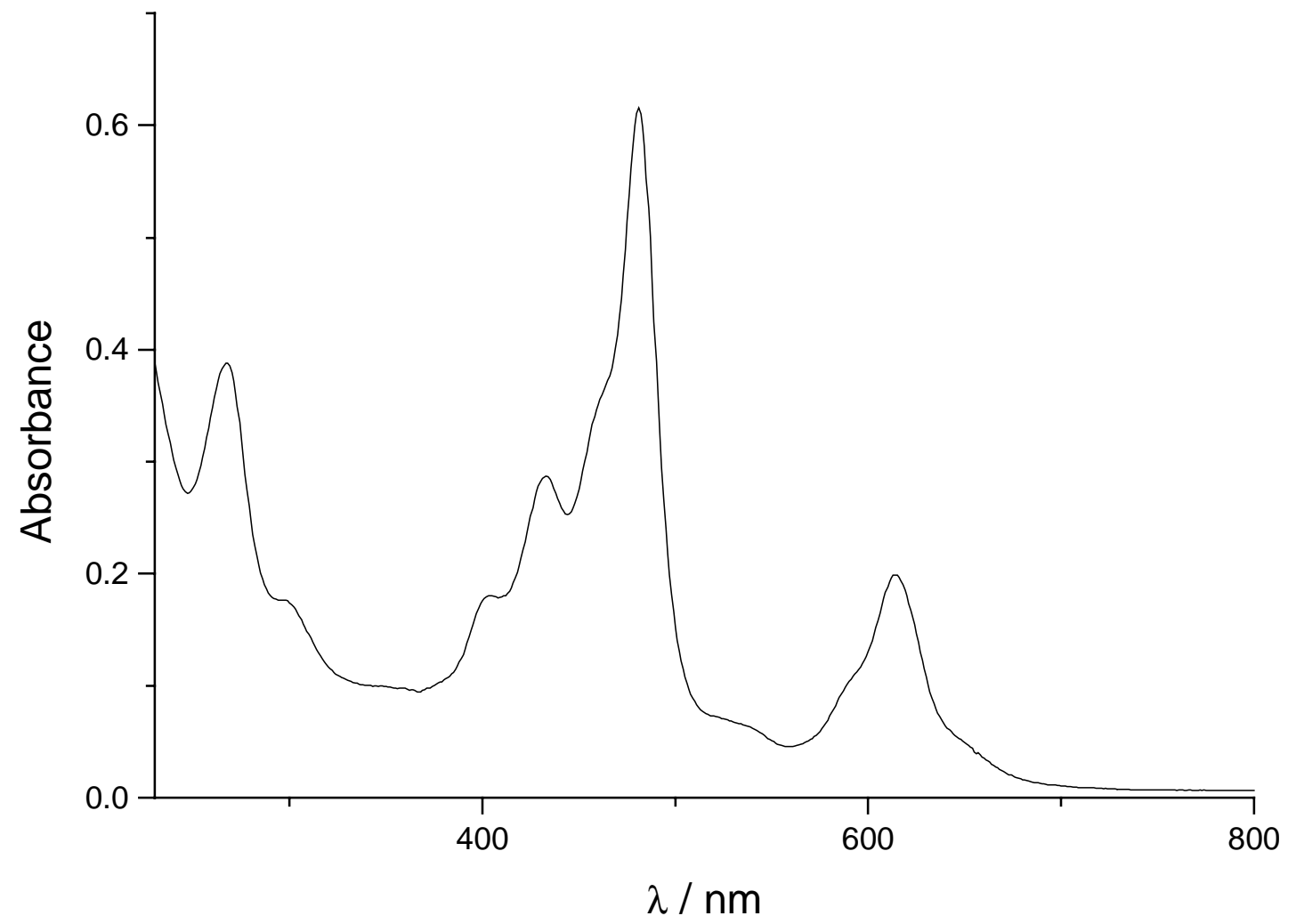

Fig. S31. Optical spectrum of $\mathbf{1 1}$ in dichloromethane. 